\title{
Higher-order dissipation in the theory of homogeneous isotropic turbulence
}

\author{
Norbert Peters ${ }^{1}$, Jonas Boschung ${ }^{1} \uparrow$, Michael Gauding 2 , \\ Jens Henrik Goebbert ${ }^{3}$, Reginald J. Hill ${ }^{4}$ and Heinz Pitsch ${ }^{1}$ \\ ${ }^{1}$ Institute for Combustion Technology, RWTH Aachen University, Templergraben 64, \\ 52056 Aachen, Germany \\ ${ }^{2}$ Chair of Numerical Thermo-Fluid Dynamics, TU Bergakademie, Fuchsmühlenweg 9, \\ 09599 Freiberg, Germany \\ ${ }^{3}$ Jülich Supercomputing Centre, Forschungzentrum Jülich GmbH, Wilhelm-Johnen-Strasse, \\ 52425 Jülich, Germany \\ ${ }^{4} 935$ Yale Road, Boulder, CO 80305, USA
}

(Received 22 May 2015; revised 19 July 2016; accepted 20 July 2016; first published online 19 August 2016)

The two-point theory of homogeneous isotropic turbulence is extended to source terms appearing in the equations for higher-order structure functions. For this, transport equations for these source terms are derived. We focus on the trace of the resulting equations, which is of particular interest because it is invariant and therefore independent of the coordinate system. In the trace of the even-order source term equation, we discover the higher-order moments of the dissipation distribution, and the individual even-order source term equations contain the higher-order moments of the longitudinal, transverse and mixed dissipation distribution functions. This shows for the first time that dissipation fluctuations, on which most of the phenomenological intermittency models are based, are contained in the Navier-Stokes equations. Noticeably, we also find the volume-averaged dissipation $\varepsilon_{r}$ used by Kolmogorov (J. Fluid Mech., vol. 13, 1962, pp. 82-85) in the resulting system of equations, because it is related to dissipation correlations.

Key words: isotropic turbulence, mathematical foundations, turbulent flows

\section{Introduction}

Fully developed Navier-Stokes turbulence at high Reynolds numbers is characterized by a large range of length scales, varying from the geometrical lengths of the flow over the so-called integral length scale $L$, at which large velocity fluctuations occur on average, down to the Kolmogorov or dissipation scale $\eta$, at which kinetic energy is dissipated. While flow properties at the geometrical and integral length scales are influenced by boundary conditions, it is believed that fluctuations at the small scales are approximately isotropic in a statistical sense. In order to study these fluctuations, one focuses on an idealized flow, the so-called homogeneous isotropic turbulence, where all mean velocities are zero. This case may then be treated by

$\dagger$ Email address for correspondence: j.boschung@itv.rwth-aachen.de 
methods of statistical physics, conditioned on strictly satisfying the Navier-Stokes equations. Statistics can be described by two-point equations for statistical moments of velocity fluctuations, which contain the mean dissipation $\langle\varepsilon\rangle$ at the second order. The understanding is that there is a steady cascade of kinetic energy from the large scales to the small scales, with $\langle\varepsilon\rangle$ being equal to the rate of energy transfer from the large scales to the small scales. Hence, $\langle\varepsilon\rangle$ may be determined at the large scales, essentially at the integral scale $L$, and therefore is an external parameter imposed on the two-point equations describing small-scale turbulence.

The basis of this theory was laid by two papers of Kolmogorov in 1941. In Kolmogorov (1941b) he described velocity fluctuations separated by a distance $r$ as two-point velocity differences or velocity increments, the statistical moments of which are known as structure functions. He then introduced the dissipation scale $\eta$, which essentially subdivides the range of small-scale turbulence into two subranges, a dissipative subrange for small $r$, where both the viscosity $v$ and the dissipation $\langle\varepsilon\rangle$ determine the solution of the second-order structure function equation, and an inertial range for large $r$, where only $\langle\varepsilon\rangle$ remains as a scaling parameter. In Kolmogorov (1941a) he presented, based on the 'known equation of von Kármán for the isotropic turbulence in the sense of Taylor', an exact ordinary differential equation for the second-order structure function, in which the distance $r$ between the two points is the independent variable. Kolmogorov (1941a) presented two separate asymptotic solutions, one for the viscous range, defined by the asymptotic limit $r \rightarrow 0$, where the viscous term and the source term balance, and another one for the inertial range known as the 4/5 law, where the transport term balances the dissipation term. The inertial range is defined as the range of large $r$ where the $4 / 5$ law is valid. The two papers Kolmogorov $(1941 a, b)$ are known as K41 in the literature. These two results are of high importance, because they are both exact (under the assumptions of (local) isotropy, (local) homogeneity and very large Reynolds numbers) and were derived from the governing Navier-Stokes equations, i.e. they are solutions of the second-order structure function equations. One of the main aims of the present paper is to analyse the fourth-order structure function equations in this spirit with emphasis on their source terms. The notion is that solutions for the higher-order structure functions must be contained in the resulting system of equations.

Kolmogorov (1941b) had postulated that $v$ and $\langle\varepsilon\rangle$ are the only scaling parameters for the entire distribution function of two-point velocity differences. Because only two quantities with different physical units are needed to non-dimensionalize the structure function equations, this was viewed as a claim for universality. However, Landau has argued that universality would be violated by variations of the dissipation at the large scales (cf. Landau \& Lifshitz 1959; Frisch 1995). To address Landau's criticism, Obukhov (1962) suggested the replacement of $\langle\varepsilon\rangle$ by a local average $\varepsilon_{r}$ over a volume of size $r$, where $\varepsilon_{r}$ is a fluctuating quantity, and assumed that the dissipation was log-normally distributed. Kolmogorov (1962) then quantified the variance of $\varepsilon_{r}$ and used this concept to predict the inertial-range scaling exponents $\zeta_{n, 0}$ defined by

$$
S_{n, 0}=\left\langle\left[u_{1}\left(x_{1}+r, x_{2}, x_{3}\right)-u_{1}\left(x_{1}, x_{2}, x_{3}\right)\right]^{n}\right\rangle \propto r^{\zeta_{n, 0}},
$$

where $\boldsymbol{u}$ is the velocity field and $S_{n, 0}$ is the longitudinal velocity structure function of order $n$. While in K41 the inertial-range scaling exponents had been $\zeta_{n, 0}=n / 3$, Kolmogorov (1962) predicted deviations from the K41 scaling, referred to as anomalous scaling in the literature, which are described by a single parameter, the intermittency coefficient $\mu$.

The two papers Kolmogorov (1962) and Obukhov (1962) are known as K62 in the literature, or as the refined similarity hypothesis ( $\mathrm{RSH})$. While $\mathrm{K} 41$ is based on 
the Navier-Stokes equations, the RSH remains a phenomenological model with no apparent connection to the Navier-Stokes equations. However, since dissipation fluctuations must be contained in the Navier-Stokes equations, the parameters describing them should be hidden somewhere in the equations for the higher-order structure functions or in additional equations related to them. More specifically, the moments of the dissipation distribution function should appear in averaged two-point equations derived from the Navier-Stokes equations. We will show in this paper that moments of specific forms of dissipation do indeed appear in additional equations to be derived. Hereafter, we will refer to these moments as dissipation parameters. Below, we find that the approach of using $\varepsilon_{r}$ is consistent with the two-point theory of small-scale turbulence in the sense that $\varepsilon_{r}$ appears in the system of the newly derived equations. However, the relation of the ansatz $\left\langle\varepsilon_{r}^{n / 3}\right\rangle$ for the $n$ th-order structure function to the Navier-Stokes equations is still missing.

There have been various attempts to modify the RSH by proposing other functions than the log-normal distribution function. Some examples are the multifractal model by Meneveau \& Sreenivasan (1991), or the She \& Lévêque (1994) model, which later was shown to be equivalent to assuming a log-Poisson distribution for the dissipation, cf. Dubrulle (1994) and She \& Waymire (1995). A discussion of these models is given by Frisch (1995). A survey of the work existing in 1997 on the RSH, anomalous scaling exponents, velocity derivative statistics and intermittency models was given by Sreenivasan \& Antonia (1997).

The basis of our analysis lies in the exact equations for structure functions of all orders which Hill (2001) and Yakhot (2001) derived from the Navier-Stokes equations. Other than in the trace of the second-order equation, the source terms in the higher-order equations depend on $r$ and contain correlations between pressure gradients, velocity gradients squared and velocity differences. The source terms containing pressure gradients will be called pressure source terms and those containing velocity gradients will be called dissipation source terms in the following.

The papers of Hill (2001) and Yakhot (2001) differ substantially (cf. http://arxiv.org/abs/physics/0102055). Hill (2001) derived the structure function equations using no approximations, then simplified them for the anisotropic locally homogeneous and then to the (locally) homogeneous isotropic case. For the latter he invented a matrix algorithm to generate expressions for the isotropic forms for the transport terms and the viscous terms for all orders $n$ from 2 to 7 . By using isotropic tensor relations, he identified the non-zero and zero components of the tensors and derived kinematic relationships between the non-zero components. Yakhot (2001) started his derivation from a generating function and allowed for arbitrary dimensions. Assuming locally homogeneous and isotropic turbulence, the structure functions can be generated by successive differentiation. For the three-dimensional case, he considered the equations of uneven order (which contain the even-order structure function in the transport terms) and neglected the dissipation source terms therein. For the pressure source terms, he derived a model using the probability density function of the transverse velocity increments. The purpose of Yakhot's paper was to derive a formula for the transverse inertial-range scaling exponents of even order. More recently, Falkovich, Fouxon \& Oz (2010) started from a general flux equation from which they derived an analogue to Kolmogorov's 4/5 law for compressible turbulence, as well as a new fifth-order relation for incompressible turbulence. L'vov \& Procaccia (1996b) considered multipoint correlation functions for which they formulated fusion rules and used correlations between the one-point dissipation rates and velocity differences to relate the scaling exponents of structure functions and 
dissipation fluctuations. The multipoint approach by L'vov \& Procaccia (1996b) can be viewed as a generalization of the classical two-point statistics, as the latter are contained in the former by fusion of the coordinates. Fusion rule predictions were examined by Fairhall et al. (1997) and Benzi, Biferale \& Toschi (1998) and found to be in good agreement with measurements. In principle, it is possible to derive transport equations for the general multipoint quantities and their source terms using the procedure of Hill (2001). However, these equations are somewhat cumbersome to derive and to examine. For that reason, here we rather focus on the classical two-point structure functions characterized by only one separation distance and not two or more, thus simplifying the analysis.

In the literature, source terms of higher-order structure function equations have been analysed by direct numerical simulations (DNS) and, to the extent that this was possible, by hot-wire measurements. Hill \& Boratav (2001) analysed the third-order structure function equations based on DNS and experiments. From their analysis, it appears that only the pressure source terms determine the solutions at the third order. Kurien \& Sreenivasan (2001) discussed the Yakhot (2001) paper and the models presented therein in detail. They then used high-Reynolds-number experimental data from the atmospheric boundary layer to compute the pressure terms from Yakhot's model and balance the terms of the transverse and mixed fourth-order structure function equations in the inertial range. Gotoh \& Nakano (2003) have examined on the basis of DNS data the balances between the even-order structure functions and the pressure source terms in the uneven-order equations up to eighth order and proposed a model for the pressure source terms. Yakhot (2003) has modified this model to obtain the same formula as in Yakhot (2001), but now for longitudinal inertial-range scaling exponents. Based on a model for the probability density function for longitudinal velocity increments, Yakhot (2006) discussed the closure of the structure function equations in terms of the dissipation anomaly (cf. Polyakov 1995; Yakhot \& Sreenivasan 2005). Yakhot (2006) also introduced order-dependent dissipation scales $\eta_{n}$. Using DNS data, Nakano, Gotoh \& Fukayama (2003) normalized the dissipation source terms in the longitudinal equations up to eighth order by the next-order structure function. They found that for all even-order equations, the normalized dissipation source terms are of order unity. This suggests that the dissipation source terms rather than the pressure source terms are dominant in the even-order equations.

Here, we will focus on the trace of the fourth-order structure function equations rather than the component equations, because it simplifies the treatment of the equations. Additionally, there is an analogy to the trace of the second-order structure function equations, which contains the mean dissipation $\langle\varepsilon\rangle$. We may therefore expect to find the second moment $\left\langle\varepsilon^{2}\right\rangle$ in the fourth-order equations. In this context, it is also worth mentioning that the trace is of particular interest because it is invariant, i.e. independent of the coordinate system, as are $\langle\varepsilon\rangle$ and Siggia (1981)'s invariants. This is especially important if one is interested in scaling parameters such as $\left\langle\varepsilon^{m}\right\rangle$. For instance, Hill (2002) showed that the mean dissipation $\langle\varepsilon\rangle$ is found in the trace of the second-order structure function equations (those quantities being invariants), while the equations for the components of the second-order structure function contain the pseudo-dissipation tensor $\left\langle e_{i j}\right\rangle$. Kolmogorov's K41 equation relating a component of the third-order structure function to the invariant $\langle\varepsilon\rangle$ is obtained because of the incompressibility relation (cf. (3.22)-(3.23) in Hill (2002)). Analogous incompressibility relations do not exist at higher order, such that one should not expect equations relating individual components of higher-order structure functions to an invariant quantity analogous to $\langle\varepsilon\rangle$. 
The paper is organized as follows. In $\S 2$, we will present the equation for the trace of the fourth-order structure functions and the equation for the dissipation source term therein. We will find in the source term equations several new dissipation parameters. In $\S 3$, we will relate these dissipation parameters to each other and to the second moment $\left\langle\varepsilon^{2}\right\rangle$ of the distribution function of the dissipation $\varepsilon$. We will also show how they are related to the fourth-order moments of the velocity gradient distribution function and to the solutions of the fourth-order structure functions in the viscous range. In $\S 4$, we will investigate the effect of dissipation parameters on the inertial-range scaling exponents by integrating the structure function equations in the inertial range. Using order-of-magnitude estimates for the different source terms, we thereby determine the fifth-order scaling exponents. We will also look at the connection between dissipation fluctuations traditionally represented by the volume-averaged dissipation $\varepsilon_{r}$ and the dissipation parameters identified in the dissipative source term equations. Conclusions are briefly presented in $\S 5$.

\section{Equations for the trace of structure functions and dissipation source terms at the fourth-order level}

With the procedure used by Hill (2001), we have derived equations for successive source terms appearing in the equations for structure functions up to the sixth order under the assumptions of homogeneity and isotropy. The idea is that the source terms determine the solution of the structure function equations, i.e. the structure functions themselves. In the following, we present the equations used in the next sections. The entire set of successive equations (up to the sixth order) is documented in the archive material available from http://arxiv.org/abs/1504.07490. While it is possible to carry out the same analysis at higher orders, both the number of equations and the number of terms to be analysed increase very quickly; for that reason we consider only the fourth order here. We will look at the fourth-order equations in the viscous range in $\S 3$, while the inertial-range assumptions are used in $\S 4$. We have also performed DNS in a cubic box for Taylor-based Reynolds numbers up to 529 and for some parts of the analysis up to $R e_{\lambda}=754$. This is briefly described in appendix A.

The two points at which velocity fluctuations are sampled are denoted as $\boldsymbol{x}=\left(x_{1}, x_{2}, x_{3}\right)$ and $\boldsymbol{x}^{\prime}=\left(x_{1}^{\prime}, x_{2}^{\prime}, x_{3}^{\prime}\right)$, with $x_{1}$ pointing in the longitudinal direction and $x_{2}$ and $x_{3}$ in the transverse direction. Structure functions are defined as $S_{p, q}=\left\langle\left(\Delta u_{1}\right)^{p}\left(\Delta u_{2}\right)^{q}\right\rangle$, with $\Delta u_{1}=u_{1}-u_{1}^{\prime}$ being the longitudinal and $\Delta u_{2}=u_{2}-u_{2}^{\prime}$ the transverse velocity difference, with $u_{1}$ and $u_{2}$ taken at $x_{1}$ and $u_{1}^{\prime}$ and $u_{2}^{\prime}$ at $x_{1}^{\prime}$. For the $n$ th-order moment, $n=p+q$ in these equations. Hence, at the fourth order, $S_{4,0}$ is the longitudinal, $S_{2,2}$ is the mixed and $S_{0,4}$ is the transverse structure function. In the following, Einstein's summation convention is used, which implies summation over indices appearing twice. For instance, $\left\langle\varepsilon_{i j}^{2}\right\rangle=\left\langle\varepsilon_{i j} \varepsilon_{i j}\right\rangle$, where the sum is over $i$ and $j$. The equations for $S_{4,0}, S_{2,2}$ and $S_{0,4}$ are given in appendix B. In this paper, we examine the trace of the structure function tensor

$$
S_{[4]}=\left\langle\Delta u_{i} \Delta u_{i} \Delta u_{j} \Delta u_{j}\right\rangle,
$$

and the same analysis can be carried over to the individual equations. As

$$
\left.\begin{array}{c}
\left\langle\left(\Delta u_{1}\right)^{2}\left(\Delta u_{2}\right)^{2}\right\rangle=\left\langle\left(\Delta u_{1}\right)^{2}\left(\Delta u_{3}\right)^{2}\right\rangle, \\
\left\langle\left(\Delta u_{2}\right)^{4}\right\rangle=3\left\langle\left(\Delta u_{2}\right)^{2}\left(\Delta u_{3}\right)^{2}\right\rangle=\left\langle\left(\Delta u_{3}\right)^{4}\right\rangle,
\end{array}\right\}
$$

cf. Hill (2001), the trace amounts to the sum

$$
S_{[4]}=S_{4,0}+4 S_{2,2}+\frac{8}{3} S_{0,4} \text {. }
$$


Consequently, the transport equation for $S_{[4]}$ is derived by adding the individual structure function equations (B 1)-(B 3) as given in appendix B multiplied by the corresponding prefactor. This results in

$$
\frac{\partial S_{[4]}}{\partial t}+\frac{\partial}{\partial r_{n}}\left\langle\Delta u_{n}\left(\Delta u_{i}\right)^{2}\left(\Delta u_{j}\right)^{2}\right\rangle=2 v \frac{\partial^{2} S_{[4]}}{\partial r_{n}^{2}}-\left\langle T_{[4]}\right\rangle-\left\langle E_{[4]}\right\rangle .
$$

Here, the second term on the left-hand side is the transport term containing the fifth-order longitudinal, mixed and transverse structure functions $S_{5,0}, S_{3,2}$ and $S_{1,4}$ respectively. The first term on the right-hand side of (2.4) is the viscous term containing the fourth-order structure functions $S_{4,0}, S_{2,2}$ and $S_{0,4}$. The remaining two terms in the equation are the pressure source term and the dissipative source term in that order. Equation (2.4) is closely related to equation (4.3) of Falkovich et al. (2010) (also a fourth-order equation), which they derived from the kinetic energy equation. From this, they found a new relation for the pressure-velocity correlation in the inertial range, similar to Kolmogorov's 4/5 law stemming from the second order.

In explicit notation, the transport term is written as

$$
\begin{aligned}
\frac{\partial}{\partial r_{n}}\left\langle\Delta u_{n}\left(\Delta u_{i}\right)^{2}\left(\Delta u_{j}\right)^{2}\right\rangle & =\frac{\partial S_{5,0}}{\partial r}+4 \frac{\partial S_{3,2}}{\partial r}+\frac{8}{3} \frac{\partial S_{1,4}}{\partial r}+\frac{2}{r} S_{5,0}+\frac{8}{r} S_{3,2}-\frac{16}{3 r} S_{1,4} \\
& =\frac{\partial S_{[5]}}{\partial r}+\frac{2}{r} S_{[5]},
\end{aligned}
$$

where $S_{[5]}$ is the trace of the general fifth-order structure function tensor,

$$
S_{[5]}=\left\langle\Delta u_{1}\left(\Delta u_{i}\right)^{2}\left(\Delta u_{j}\right)^{2}\right\rangle=S_{5,0}+4 S_{3,2}+\frac{8}{3} S_{1,4} .
$$

The pressure source term is given by

$$
\begin{aligned}
\left\langle T_{[4]}\right\rangle= & 4\left\langle\left(\Delta u_{i}\right)^{2} \Delta u_{j} \Delta P_{j}\right\rangle \\
= & 4\left\langle\left(\Delta u_{1}\right)^{3} \Delta P_{1}\right\rangle+8\left\langle\left(\Delta u_{2}\right)^{2} \Delta u_{1} \Delta P_{1}+\left(\Delta u_{1}\right)^{2} \Delta u_{2} \Delta P_{2}\right\rangle \\
& +\frac{32}{3}\left\langle\left(\Delta u_{2}\right)^{3} \Delta P_{2}\right\rangle,
\end{aligned}
$$

the dissipative source term by

$$
\begin{aligned}
\left\langle E_{[4]}\right\rangle= & \left\langle 2\left(\Delta u_{i}\right)^{2}\left(\varepsilon_{j j}+\varepsilon_{j j}^{\prime}\right)+4 \Delta u_{i} \Delta u_{j}\left(\varepsilon_{i j}+\varepsilon_{i j}^{\prime}\right)\right\rangle \\
= & 6\left\langle\left(\Delta u_{1}\right)^{2}\left(\varepsilon_{11}+\varepsilon_{11}^{\prime}\right)\right\rangle+4\left\langle\left(\Delta u_{2}\right)^{2}\left(\varepsilon_{11}+\varepsilon_{11}^{\prime}\right)+\left(\Delta u_{1}\right)^{2}\left(\varepsilon_{22}+\varepsilon_{22}^{\prime}\right)\right\rangle \\
& +16\left\langle\Delta u_{1} \Delta u_{2}\left(\varepsilon_{12}+\varepsilon_{12}^{\prime}\right)+\left(\Delta u_{2}\right)^{2}\left(\varepsilon_{22}+\varepsilon_{22}^{\prime}\right)\right\rangle
\end{aligned}
$$

and the viscous term by

$$
\begin{aligned}
2 v \frac{\partial^{2} S_{[4]}}{\partial r_{n}^{2}} & =2 v\left[\frac{\partial^{2} S_{4,0}}{\partial r^{2}}+\frac{2}{r} \frac{\partial S_{4,0}}{\partial r}+4 \frac{\partial^{2} S_{2,2}}{\partial r^{2}}+\frac{8}{r} \frac{\partial S_{2,2}}{\partial r}+\frac{8}{3} \frac{\partial^{2} S_{0,4}}{\partial r^{2}}+\frac{16}{3 r} \frac{\partial S_{0,4}}{\partial r}\right] \\
& =2 v\left[\frac{\partial^{2} S_{[4]}}{\partial r^{2}}+\frac{2}{r} \frac{\partial S_{[4]}}{\partial r}\right] .
\end{aligned}
$$

In (2.4)-(2.9), the definitions

$$
\begin{aligned}
\Delta P_{i} & =\frac{\partial p}{\partial x_{i}}-\frac{\partial p^{\prime}}{\partial x_{i}^{\prime}}, \\
\varepsilon_{i j} & =2 v \frac{\partial u_{i}}{\partial x_{k}} \frac{\partial u_{j}}{\partial x_{k}}
\end{aligned}
$$

have been used. 

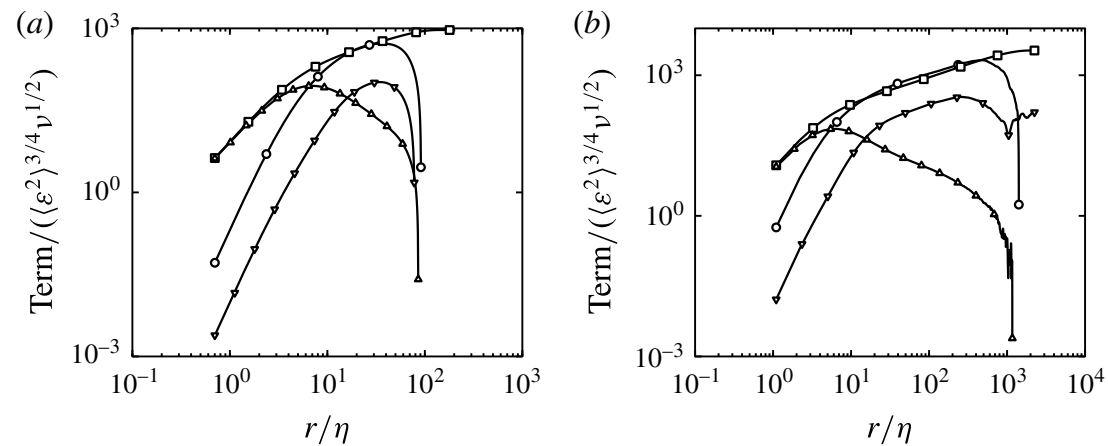

FIGURE 1. Balance of the different terms in (2.4) for the cases $R 0\left(R e_{\lambda}=88\right)(a)$ and $R 5$ $\left(\operatorname{Re}_{\lambda}=529\right)(b): \bigcirc$, transport term; $\square$, dissipative source term; $\nabla$, pressure source term; $\Delta$, viscous term.

The balance of the different terms in (2.4) is shown in figure 1(a) for the case $R 0\left(R e_{\lambda}=88\right)$ and in figure $1(b)$ for the case $R 5\left(R e_{\lambda}=529\right)$ of our DNS. We will need this balance to estimate which terms we may neglect when we integrate (2.4) in the viscous range in $\S 3$ and in the inertial range in $\S 4$ below. The terms have been normalized with $\left(v^{2}\left\langle\varepsilon^{2}\right\rangle^{3}\right)^{1 / 4}$ and the separation distance $r$ with the Kolmogorov scale $\eta$. It is seen that in the viscous range for values of $r / \eta$ up to approximately 5 , the transport term and the pressure source term are an order of magnitude smaller than the viscous terms and the dissipation source term for the six data sets $R 0-R 5$ $(R 1-R 4$ are not shown here). These terms therefore balance to leading order, which will lead to exact relations in the viscous range, as will be shown in $\S 3$. As expected, the inertial range increases with increasing Reynolds number: beginning at $r / \eta=30$, for the case $R 0\left(R e_{\lambda}=88\right)$ only a very small inertial range is identifiable, whereas for $R 5\left(R e_{\lambda}=529\right)$ it extends to $r / \eta \approx 300$. In the inertial range and for all data sets, the pressure source term is smaller by a factor of four on average and the viscous term is much smaller. This order-of-magnitude estimate will be used in $\S 4$.

The dissipation term $\left\langle E_{[4]}\right\rangle$ defined in (2.8) is a correlation between squared velocity increments $\Delta u_{1}$ and $\Delta u_{2}$ and the instantaneous dissipations defined by (2.11) at $x_{1}$ and likewise at $x_{1}^{\prime}$. Since it balances the transport term in the inertial range and the viscous term in the viscous range, $\left\langle E_{[4]}\right\rangle$ is of particular interest and will be further examined in the following. Furthermore, one might expect the dissipative source term to contain dissipative fluctuations. The equation for the dissipative source term,

$$
\left\langle E_{[4]}\right\rangle=\left\langle E_{4,0}\right\rangle+4\left\langle E_{2,2}\right\rangle+\frac{8}{3}\left\langle E_{0,4}\right\rangle,
$$

reads with the equations for $\left\langle E_{4,0}\right\rangle,\left\langle E_{2,2}\right\rangle$ and $\left\langle E_{0,4}\right\rangle$, cf. (B 10), (B 12) and (B 14) given in appendix B,

$$
\begin{aligned}
\frac{\left\langle\partial E_{[4]}\right\rangle}{\partial t}+\frac{\partial\left\langle\Delta u_{n} E_{[4]}\right\rangle}{\partial r_{n}}= & v \frac{\partial^{2}}{\partial r_{n}^{2}}\left\langle E_{[4]}\right\rangle \\
& -\underbrace{\left(F_{[4]}+Q_{[4]}+P_{[4]}+T_{[4]}+D_{[4]}+\varepsilon_{[4]}^{2}\right)}_{\sum P_{[4]}^{E}},
\end{aligned}
$$


where $\sum P_{[4]}^{E}$ is the trace of the sum of source terms in the dissipative source term equation, where the transport term is defined as

$$
\begin{aligned}
\frac{\partial\left\langle\Delta u_{n} E_{[4]}\right\rangle}{\partial r_{n}}= & \frac{\partial\left\langle\Delta u_{1} E_{4,0}\right\rangle}{\partial r}+4 \frac{\partial\left\langle\Delta u_{1} E_{2,2}\right\rangle}{\partial r}+\frac{8}{3} \frac{\partial\left\langle\Delta u_{1} E_{0,4}\right\rangle}{\partial r} \\
& +\frac{2}{r}\left\langle\Delta u_{1} E_{4,0}\right\rangle+\frac{8}{r}\left\langle\Delta u_{1} E_{2,2}\right\rangle+\frac{16}{3 r}\left\langle\Delta u_{1} E_{0,4}\right\rangle \\
= & \frac{\partial\left\langle\Delta u_{1} E_{[4]}\right\rangle}{\partial r}+\frac{2}{r}\left\langle\Delta u_{1} E_{[4]}\right\rangle,
\end{aligned}
$$

with

$$
\Delta u_{1} E_{[4]}=\Delta u_{1} E_{4,0}+4 \Delta u_{1} E_{2,2}+\frac{8}{3} \Delta u_{1} E_{0,4}
$$

the viscous term

$$
2 v \frac{\partial^{2}\left\langle E_{[4]}\right\rangle}{\partial r_{n}^{2}}=2 v\left[\frac{\partial^{2}\left\langle E_{[4]}\right\rangle}{\partial r^{2}}+\frac{2}{r} \frac{\partial\left\langle E_{[4]}\right\rangle}{\partial r}\right],
$$

the $F$-term

$$
F_{[4]}=8 v\left\langle\left(\Delta u_{i}\right)^{2}\left(A_{j j}+A_{j j}^{\prime}\right)+\Delta u_{i} \Delta u_{j}\left(A_{i j}+A_{i j}^{\prime}+A_{j i}+A_{j i}^{\prime}\right)\right\rangle,
$$

the $Q$-term

$$
Q_{[4]}=4 v\left\langle\left(\Delta u_{i}\right)^{2}\left(\chi_{j j}+\chi_{j j}^{\prime}\right)+2 \Delta u_{i} \Delta u_{j}\left(\chi_{i j}+\chi_{i j}^{\prime}\right)\right\rangle,
$$

the $P$-term

$$
P_{[4]}=4\left\langle\Delta u_{i} \Delta P_{i}\left(\varepsilon_{j j}+\varepsilon_{j j}^{\prime}\right)+\left(\Delta u_{i} \Delta P_{j}+\Delta u_{j} \Delta P_{i}\right)\left(\varepsilon_{i j}+\varepsilon_{i j}^{\prime}\right)\right\rangle,
$$

the $T$-term

$$
T_{[4]}=8 v\left\langle\left(\Delta u_{i}\right)^{2}\left(P_{j j}+P_{j j}^{\prime}\right)+\Delta u_{i} \Delta u_{j}\left(P_{i j}+P_{i j}^{\prime}+P_{j i}+P_{j i}^{\prime}\right)\right\rangle,
$$

the $D$-term

$$
\begin{aligned}
D_{[4]}= & 4 v\left\langle\left(\frac{\partial\left(\Delta u_{i}\right)^{2}}{\partial x_{n}} \frac{\partial \varepsilon_{j j}+\varepsilon_{j j}^{\prime}}{\partial x_{n}}+\frac{\partial\left(\Delta u_{i}\right)^{2}}{\partial x_{n}^{\prime}} \frac{\partial \varepsilon_{j j}^{\prime}+\varepsilon_{j j}^{\prime}}{\partial x_{n}^{\prime}}\right)\right. \\
& \left.+2\left(\frac{\partial \Delta u_{i} \Delta u_{j}}{\partial x_{n}} \frac{\partial \varepsilon_{i j}+\varepsilon_{i j}^{\prime}}{\partial x_{n}}+\frac{\partial \Delta u_{i} \Delta u_{j}}{\partial x_{n}^{\prime}} \frac{\partial \varepsilon_{i j}^{\prime}+\varepsilon_{i j}^{\prime}}{\partial x_{n}^{\prime}}\right)\right\rangle
\end{aligned}
$$

and the $\varepsilon^{2}$-term

$$
\begin{aligned}
\varepsilon_{[4]}^{2} & =\left\langle 2\left(\varepsilon_{i i}+\varepsilon_{i i}^{\prime}\right)\left(\varepsilon_{j j}+\varepsilon_{j j}^{\prime}\right)+4\left(\varepsilon_{i j}+\varepsilon_{i j}^{\prime}\right)^{2}\right\rangle \\
& =2\left\langle\left(\varepsilon+\varepsilon^{\prime}\right)^{2}\right\rangle+4\left\langle\left(\varepsilon_{i j}+\varepsilon_{i j}^{\prime}\right)^{2}\right\rangle .
\end{aligned}
$$

In our notation, $\varepsilon_{i i}=\varepsilon$ is twice the pseudo-dissipation. For better readability, the definitions

$$
\begin{gathered}
A_{i j}=\frac{\partial u_{n}}{\partial x_{m}} \frac{\partial u_{i}}{\partial x_{n}} \frac{\partial u_{j}}{\partial x_{m}}, \\
P_{i j}=\frac{\partial u_{i}}{\partial x_{m}} \frac{\partial^{2} p}{\partial x_{j} \partial x_{m}}
\end{gathered}
$$



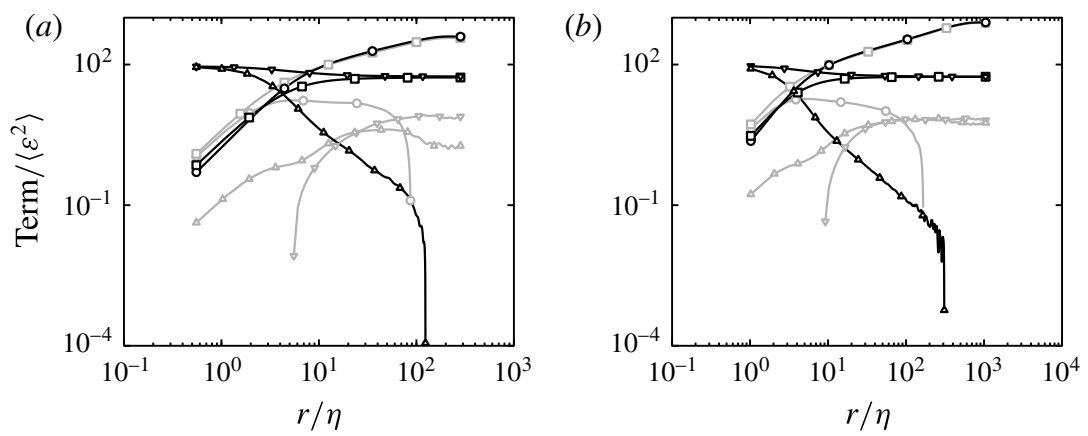

FIGURE 2. Balance of the different terms in (2.13) for the cases $R 1\left(R e_{\lambda}=119\right)(a)$ and $R 4\left(R e_{\lambda}=331\right)(b)$ : grey $O$, transport term; grey $\square, F$-term; grey $\nabla, P$-term; grey $\triangle, T$ term; $\bigcirc, Q$-term; $\square, D$-term; $\nabla, \varepsilon^{2}$-term; $\triangle$, viscous term.

and

$$
\chi_{i j}=2 v\left[\frac{\partial^{2} u_{i}}{\partial x_{n} \partial x_{m}} \frac{\partial^{2} u_{j}}{\partial x_{n} \partial x_{m}}\right]
$$

have been used in (2.17)-(2.20).

For the stationary case, the transport term on the left-hand side in (2.13) balances the viscous term and the sum of source terms $\sum P_{[4]}^{E}$. The source terms are defined in (2.17) to (2.22) and are shown in figure 2 for the cases $R 1\left(R e_{\lambda}=119\right)$ and $R 4$ $\left(R e_{\lambda}=331\right)$ together with the transport term and the viscous term. Negative terms are denoted with a minus sign, meaning that we have changed their sign in order to be able to show them in a $\log -\log$ plot. As seen in figure 2, the largest ones are the $F$-term and the $Q$-term, which nearly balance each other. The $F$-term is a correlation between the velocity increments squared and the triple product of velocity gradients $\left(A_{i j}+A_{i j}^{\prime}\right)$. The $Q$-term is a correlation between the velocity increments and $\left(\chi_{i j}+\chi_{i j}^{\prime}\right)$. The term $\chi_{i j}$ describes the dissipation of velocity gradients squared, while $A_{i j}$ describes their production by stretching. The next two source terms, called the $T$-term and the $P$-term, containing pressure derivatives, are relatively small and will not be discussed here in detail. Finally, there are the $D$-term and the $\varepsilon^{2}$-term, which balance each other in the inertial range, but strongly diverge from each other in the viscous range. In the following, the focus will be on the $\varepsilon^{2}$-term, which is the sum of the second-order dissipation parameters.

The $\varepsilon^{2}$-term is defined as $2\left\langle\left(\varepsilon_{i i}+\varepsilon_{i i}^{\prime}\right)^{2}\right\rangle+4\left\langle\left(\varepsilon_{i j}+\varepsilon_{i j}^{\prime}\right)^{2}\right\rangle$ and is a two-point quantity depending on $r$. Noticeably, Hill (2002) showed that the sum of the two-point dissipation $\left\langle\varepsilon+\varepsilon^{\prime}\right\rangle$ also appears in the trace of the second-order structure function equations, which then reduces to $2\langle\varepsilon\rangle$ independent of $r$ for homogeneous flows.

Since $\varepsilon_{i j}$ becomes equal to $\varepsilon_{i j}^{\prime}$ at $r \rightarrow 0, \varepsilon_{[4]}^{2}$ approaches the value $8\left\langle\varepsilon_{i i}^{2}\right\rangle+16\left\langle\varepsilon_{i j}^{2}\right\rangle$ and balances the viscous term there, as seen in figure 2. The quantity $\left\langle\varepsilon_{i i}^{2}\right\rangle=\left\langle\varepsilon^{2}\right\rangle$ is the second-order moment of the dissipation distribution of $\varepsilon$. It is a sum of dissipation parameters that appear in the set of successive equations presented in the archive. It is worth noting that the $\varepsilon^{2}$-term in figure 2 starts as a constant for small values of $r$, then decreases slightly and becomes constant again at the large scales. In the latter range, $2\left\langle\left(\varepsilon_{i i}+\varepsilon_{i i}^{\prime}\right)^{2}\right\rangle+4\left\langle\left(\varepsilon_{i j}+\varepsilon_{i j}^{\prime}\right)^{2}\right\rangle$ approaches the asymptotic limit $4\left\langle\varepsilon_{i i}^{2}\right\rangle+8\left\langle\varepsilon_{i j}^{2}\right\rangle$; i.e. it differs from the value for $r \rightarrow 0$ only by a constant factor of 2 . That is, the $\varepsilon^{2}$-term links the remaining terms of (2.13) at the very large and very small scales, similarly to 
$\langle\varepsilon\rangle$ at the second order. In the following, we are mostly interested in the influence of the $\varepsilon^{2}$-term, since this term is closest to the classical picture of dissipative fluctuations in turbulent flows in the spirit of Kolmogorov.

3. Relations between the trace of fourth-order structure functions $S_{[4]}$ and $\left\langle\varepsilon_{[4]}^{2}\right\rangle$ in the viscous range

First, we examine the equations outlined above in the viscous range, before we look at the inertial range in the next section. We find that for $r \rightarrow 0, S_{[4]}$ is exactly determined by $\varepsilon_{[4]}^{2}, v$ and $r$.

In the viscous range, the transport term of the fourth-order structure function (2.4) as well as the pressure source terms are proportional to $r^{4}$ for $r \rightarrow 0$, while the viscous and the dissipative source terms are proportional to $r^{2}$. Therefore, to leading order the transport and pressure source terms may be neglected in the viscous range and (2.4) simplifies to

$$
2 v\left(\frac{\mathrm{d}^{2} S_{[4]}}{\mathrm{d} r^{2}}+\frac{2}{r} \frac{\mathrm{d} S_{[4]}}{\mathrm{d} r}\right)=\left\langle E_{[4]}\right\rangle .
$$

Similarly, from the equation for $\left\langle E_{[4]}\right\rangle$, the viscous and $\varepsilon^{2}$-term balance,

$$
2 v\left(\frac{\mathrm{d}^{2}\left\langle E_{[4]}\right\rangle}{\mathrm{d} r^{2}}+\frac{2}{r} \frac{\mathrm{d}\left\langle E_{[4]}\right\rangle}{\mathrm{d} r}\right)=\varepsilon_{[4]}^{2},
$$

where $\varepsilon_{[4]}^{2}$ is constant in the viscous range. Solving (3.2) and inserting the result into (3.1) then gives

$$
S_{[4]}=\frac{\varepsilon_{[4]}^{2}}{480 v^{2}} r^{4}, \quad\left\langle E_{[4]}\right\rangle=\frac{\varepsilon_{[4]}^{2}}{12 v} r^{2} .
$$

Since $S_{[4]}$ is the sum of $S_{4,0}, S_{2,2}$ and $S_{0,4}$, all of which are also proportional to $r^{4}$ for $r \rightarrow 0$, the individual structure functions are also determined by $\varepsilon_{[4]}^{2}$ in the viscous range. In particular, by expanding the structure functions as

$$
\begin{gathered}
S_{4,0}=F_{1} r^{4}+\cdots=\left\langle\left(\frac{\partial u_{1}}{\partial x_{1}}\right)^{4}\right\rangle r^{4}+\cdots, \\
S_{2,2}=F_{2} r^{4}+\cdots=\left\langle\left(\frac{\partial u_{1}}{\partial x_{1}}\right)^{2}\left(\frac{\partial u_{2}}{\partial x_{1}}\right)^{2}\right\rangle r^{4}+\cdots, \\
S_{0,4}=F_{3} r^{4}+\cdots=\left\langle\left(\frac{\partial u_{2}}{\partial x_{1}}\right)^{4}\right\rangle r^{4}+\cdots,
\end{gathered}
$$

one can relate $\varepsilon_{[4]}^{2} / v^{2}$ to the sum of $F_{1}, F_{2}$ and $F_{3}$. Siggia (1981) has identified the four invariants of the fourth-order velocity gradient tensor as

$$
I_{1} \equiv\left\langle s^{4}\right\rangle, \quad I_{2} \equiv\left\langle s^{2} \omega^{2}\right\rangle, \quad I_{3} \equiv\left\langle\omega_{i} s_{i j} \omega_{k} s_{k j}\right\rangle, \quad I_{4} \equiv\left\langle\omega^{4}\right\rangle,
$$

where $s_{i j}=\left(\partial u_{i} / \partial x_{j}+\partial u_{j} / \partial x_{i}\right) / 2$ is the rate of strain tensor and the vorticity $\omega_{i}=$ $\epsilon_{i j k}\left(\partial u_{k} / \partial x_{j}\right)$, where $\epsilon_{i j k}$ is the Levi-Civita symbol. Siggia then derived

$$
F_{1}=4 I_{1} / 105
$$




$$
\begin{gathered}
F_{2}=I_{1} / 105+I_{2} / 70-I_{3} / 105, \\
F_{3}=3 I_{1} / 140+11 I_{2} / 140-3 I_{3} / 35+I_{4} / 80,
\end{gathered}
$$

resulting in

$$
\frac{\varepsilon_{[4]}^{2}}{480 v^{2}}=F_{1}+4 F_{2}+\frac{8}{3} F_{3}=\frac{2}{15} I_{1}+\frac{4}{15} I_{2}-\frac{4}{15} I_{3}+\frac{1}{30} I_{4} .
$$

Since

$$
\frac{\left\langle\varepsilon_{11}^{2}\right\rangle}{v^{2}}=\frac{\left\langle\varepsilon_{22}^{2}\right\rangle}{v^{2}}=\frac{\left\langle\varepsilon_{11} \varepsilon_{22}\right\rangle+2\left\langle\varepsilon_{12}^{2}\right\rangle}{v^{2}}=\frac{8}{15} I_{1}+\frac{16}{15} I_{2}-\frac{16}{15} I_{3}+\frac{2}{15} I_{4},
$$

cf. (C 20) in appendix $\mathrm{C}$, the trace $\varepsilon_{[4]}^{2}$ is related in the viscous range to the dissipation parameters

$$
\varepsilon_{[4]}^{2}=120\left\langle\varepsilon_{11}^{2}\right\rangle=120\left\langle\varepsilon_{22}^{2}\right\rangle=120\left\langle\varepsilon_{11} \varepsilon_{22}\right\rangle+360\left\langle\varepsilon_{12}^{2}\right\rangle .
$$

A similar analysis can be carried out for higher even orders in the viscous range. However, there are more intermediate equations linking the higher-order moments of the dissipation to the higher-order dissipative source term and ultimately to the structure function; cf. the archive for the sixth-order equations. For instance, one then finds for the sixth order $S_{[6]} \sim r^{6} \varepsilon_{[6]}^{3} / \nu^{3}$ in the viscous range, with two intermediate equations.

\section{Relation between dissipation fluctuations and inertial-range scaling exponents}

In the inertial range, Kolmogorov (1962) included dissipation fluctuations by using a locally averaged dissipation $\varepsilon_{r}$ as the structure function scaling parameter instead of only the mean value $\langle\varepsilon\rangle$. One would therefore think that $\varepsilon_{r}$ must appear in the system of equations. For that reason, we examine in this section a connection between the $\varepsilon^{2}$-term and the second moment of $\varepsilon_{r}$. Therefore, in order to analyse the effect of the dissipation parameter $\varepsilon_{[4]}^{2}$ on the inertial-range scaling exponent $\zeta_{[5]}$, we must consider the dissipative source term equation. In a first step, we will integrate (2.4) in the inertial range in order to calculate the fifth-order scaling exponent $\zeta_{[5]}$ implicitly defined by assuming a power law $S_{[5]} \sim r^{\zeta[5]}$. The idea is that because there are equations for the structure functions as well as equations for their source terms, the scaling exponent has to be contained in the system of equations.

For some time, there has been an expectation that the connection between the Navier-Stokes equations and the RSH would be discovered one day or, as Kaneda $\&$ Morishita (2013) proffered, 'the link between these models and the Navier-Stokes dynamics governing the fluid motion appears still to be missing'. This would require that a correlation exists between $\varepsilon_{r}$ and the terms representing dissipation fluctuations in the Navier-Stokes equations. In the two-point equations derived systematically from the Navier-Stokes equations in the archive material, from which we derived (2.13) shown above, we have identified the terms describing dissipation fluctuations as $\varepsilon^{2}$-terms. They contain moments of the sum of components of the dissipation at two points, for instance the moments $\left\langle\left(\varepsilon+\varepsilon^{\prime}\right)^{m}\right\rangle$. An integral expression such as

$$
\widetilde{\varepsilon}_{r}=\frac{1}{V} \int \varepsilon(\boldsymbol{x}) \mathrm{d} \boldsymbol{x}
$$


where $V \sim O\left(r^{3}\right)$ is a volume of dimension $r^{3}$, or the corresponding one-dimensional expression

$$
\varepsilon_{r}=\frac{1}{r} \int \varepsilon(x) \mathrm{d} x
$$

where the integral is over any length $r$, does not appear directly in these equations.

Since $\varepsilon_{r}$ exhibits a power-law scaling in the inertial range, dimensional analysis suggests that this could be related to the power-law scaling of the structure functions, as Kolmogorov (1962) had predicted. Stolovitzky, Kailasnath \& Sreenivasan (1992) have performed a test of the relation between $\Delta u(r)$ and $\left(r \varepsilon_{r}\right)^{1 / 3}$ by investigating the statistical properties of $\Delta u(r) /\left(r \varepsilon_{r}\right)^{1 / 3}$. They found that the distribution function of $\Delta u(r) /\left(r \varepsilon_{r}\right)^{1 / 3}$ is independent of $r$ and $\varepsilon_{r}$ in the inertial range and that it is approximately universal. Additional work addressing tests of the RSH is referenced by Sreenivasan \& Antonia (1997).

On the other hand, properties such as inertial-range scaling exponents should, as a matter of principle, result from the two-point equations. If a power-law scaling can be assumed for the source terms in the structure function equations, the scaling exponents would follow from equations like (4.7) below, to which both the pressure source term and the dissipation source term contribute. For even-order scaling exponents resulting from the odd-order structure function equations, Yakhot (2003) has argued that the dissipation source terms can be neglected and has proposed an algebraic closure relating the pressure source terms of arbitrary order to the structure functions. A similar closure could conceivably be developed for the odd-order scaling exponents in the even-order structure function equations. In these equations, the closure would be between the dissipation source terms and structure functions, while the pressure source terms can be neglected. The two-point equations show that dissipation fluctuations are represented by the dissipation parameters appearing in the successive source term equations and not by the source terms in the structure function equations. If one wanted to reconcile the $\mathrm{RSH}$ with the two-point equations, one would have to develop a closure between the moments of $\varepsilon_{r}$ and the dominating source terms in the structure function equations.

It can be shown that the second moment of $\varepsilon_{r}$ is related to the dissipation correlation $\left\langle\varepsilon \varepsilon^{\prime}\right\rangle$ under the assumption of homogeneity by

$$
\left\langle\varepsilon \varepsilon^{\prime}\right\rangle=\frac{1}{2} \frac{\mathrm{d}^{2}}{\mathrm{~d} r^{2}}\left[r^{2}\left\langle\varepsilon_{r}^{2}\right\rangle\right],
$$

cf. Nelkin (1994), and similar relations can be found for the higher moments of $\varepsilon_{r}$. We only discuss the second moment, because we examine here the fourth-order structure function equations. In particular, we only look at the trace (2.13), but our findings carry over to the individual equations for longitudinal, mixed and transverse dissipative source terms as well. If one assumes a power law for $\left\langle\varepsilon_{r}^{2}\right\rangle \sim r^{\gamma}$, where the scaling exponent and the prefactor are independent of $r$, as one is apt to do and which is at the very core of the $\mathrm{RSH}$ and similar theories, one also finds by virtue of (4.3) that $\left\langle\varepsilon \varepsilon^{\prime}\right\rangle \sim r^{\gamma}$ and hence has the same $r$-scaling. The first term of the $\varepsilon^{2}$-term in (2.22) can be written as the sum of constants and correlations; i.e.

$$
\left\langle\left(\varepsilon+\varepsilon^{\prime}\right)^{2}\right\rangle=2\left\langle\varepsilon^{2}\right\rangle+2\left\langle\varepsilon \varepsilon^{\prime}\right\rangle .
$$

In other words, the $r$-dependence of $\varepsilon_{r}$ is manifested in the $\varepsilon^{2}$-terms in the dissipative source terms by virtue of (4.3) and (4.4). 
We begin with the integration of (2.4) to link the structure function exponent to the source terms. We will neglect the diffusive and unsteady terms in (2.4) and (2.13) and perform an order-of-magnitude estimate of the source terms. If we assume in (2.4) a power-law scaling for the source terms of the form

$$
\begin{aligned}
& \left\langle T_{[4]}\right\rangle=A_{[4]}^{T} r^{\xi^{T}}{ }^{T / 4]}, \\
& \left\langle E_{[4]}\right\rangle=A_{[4]}^{E} r^{\xi_{[4]}^{E},}
\end{aligned}
$$

which is consistent with figure 1 , the trace of the fifth-order structure functions $S_{[5]}$ can be determined from (2.4) by integration. Of course, (4.5) and (4.6) are approximations. However, without these assumptions, a power law of the form $S_{[5]}=C_{[5]} r^{\zeta[5]}$ is not compatible with (2.4). In other words, only if the source terms follow a power law in the inertial range, do the structure functions as well. The range of integration will be divided into two parts. The first part ranges from $r=0$ to $r^{*} \approx 30 \eta$, where the power law in the inertial range starts to be valid. The second part ranges from there on to the value $r$ of interest in the inertial range. The integration then yields

$$
\begin{aligned}
-S_{[5]}= & \frac{1}{r^{2}} \int_{0}^{r^{*}} r^{2}\left(\left\langle T_{[4]}\right\rangle+\left\langle E_{[4]}\right\rangle\right) \mathrm{d} r-\frac{1}{r^{2}}\left\{\frac{A_{[4]}^{T} r^{3+\xi_{[4]}^{T}}}{3+\xi_{[4]}^{T}}+\frac{A_{[4]}^{E} r^{3+\xi_{[4]}^{E}}}{3+\xi_{[4]}^{E}}\right\}_{r=r^{*}} \\
& +\frac{A_{[4]}^{T} r^{1+\xi_{[4]}^{T}}}{3+\xi_{[4]}^{T}}+\frac{A_{[4]}^{E} r^{1+\xi_{[4]}^{E}}}{3+\xi_{[4]}^{E}} .
\end{aligned}
$$

Since the diffusion terms have been neglected from the very beginning, the first integral is incomplete. The purpose here is to show that this and the second term on the right-hand side of (4.7) can be neglected for large $r \gg r^{*}$ because of the term $r^{-2}$ in front of them. Then, only the last two terms on the right-hand side of (4.7) remain, and we find the trace of the structure functions to be given as a weighted sum of the two power laws (4.5) and (4.6). More specifically, there are several possible contributions to the inertial range that have been neglected: the integral from 0 to $r$ over the viscous term might not be negligible in the inertial range at $r$; the integral of the dissipative and pressure source terms over 0 to $r^{*}$ might not be negligible in the inertial range at some $r$; the value of the transport term at $r=r^{*}$ might not be small compared with its inertial-range value. Additionally, because $\eta$ is not the correct dissipative length scale for the fourth-order equations, $r^{*} / \eta$ is dependent on the Reynolds number, and this dependence is required to be small. While the importance of some of these contributions may already be estimated from the balance figure 1 , it is more advantageous to look at the integrated balance, in the spirit of (4.7) and the present section. For that reason, the numerical integration of (2.4) over $r$ is presented in figure 3. The integrated balances for the data sets $R 0\left(R e_{\lambda}=88\right)$ and $R 5\left(R e_{\lambda}=529\right)$ normalized by $\left\langle\varepsilon^{2}\right\rangle$ and $v$ are plotted over $r / \eta$. Indeed, the integrated viscous terms are negligible compared with all other integrated terms in the inertial range after $r / \eta=30$. Moreover, the integrated dissipative source term, pressure source term and transport term are much smaller in the viscous range than in the inertial range. This holds for all data sets and not only the cases $R 0$ and $R 5$ shown in figure 3(a). Last, as shown in figure 3(b), both the integrated dissipative source terms and the integrated viscous terms cross over to the inertial range at approximately the same value of $r / \eta$ for the data sets analysed here, i.e. the true viscous cutoff length scale depends only weakly on the Reynolds number. Therefore, the simplifications 

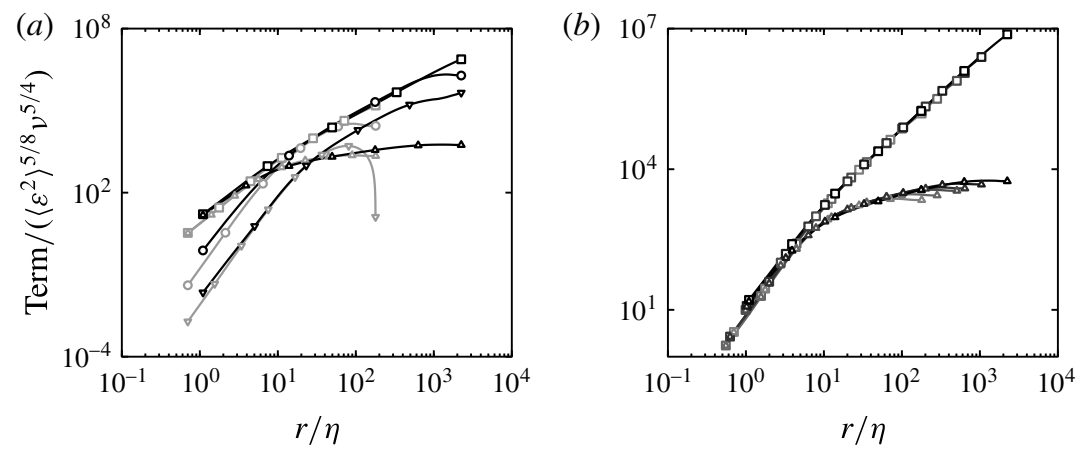

FiguRE 3. (a) Terms of (2.4) numerically integrated over $r$ for cases $R 0$ (grey) and $R 5$ (black), where $\bigcirc$ is the transport term, $\square$ is the dissipative source term, $\nabla$ is the pressure source term and $\Delta$ is the viscous term. The integrated dissipative source term $\square$ and integrated viscous terms $\Delta$ for all cases $R 0-R 5$ (from light grey to black) are shown in (b).

with regard to the integration carried out in (4.7) as described above seem to be justifiable.

The sum of two power laws with constant prefactor and exponent does not give a power law with constant prefactor and exponent. Only if $\xi_{[4]}^{T}=\xi_{[4]}^{E}$ or if one of the two terms is much smaller than the other one will the scaling of $S_{[5]}$ result in an (approximate) power law. We will explore the second possibility by approximating the scaling exponent $\zeta_{[5]}$ by neglecting the pressure source term (cf. figure 1, where the pressure source term is smaller than the dissipative source terms by a factor of four, and also figure 3), resulting in

$$
\zeta_{[5]}=1+\xi_{[4]}^{E} .
$$

This relationship between $\zeta_{[5]}$ and $\xi_{[4]}^{E}$ is consistent with fusion rules, cf. L'vov \& Procaccia (1996a) and Benzi et al. (1998), where the same relation for the fifth-order structure function is provided for the case when one of the two separation distances is in the dissipative and the other in the inertial range. Here, (4.8) follows directly from neglecting the pressure source term. We have calculated the scaling exponent $\xi_{[4]}^{E}$ of $\left\langle E_{[4]}\right\rangle$ already introduced in (4.6) for all of our DNS cases by using

$$
\xi_{[4]}^{E}=\frac{r}{\left\langle E_{[4]}\right\rangle} \frac{\partial\left\langle E_{[4]}\right\rangle}{\partial r} .
$$

As seen from figure $4, \xi_{[4]}^{E}=0.56$ in the inertial range for our data sets $R 1-R 6$. The trace of the fifth-order structure functions $S_{[5]}$ is shown in figure 5 in compensated form; i.e. divided by $(r / \eta)^{1.56}$ and normalized by $v$ and $\left\langle\varepsilon^{2}\right\rangle$. Since $\left\langle\varepsilon^{2}\right\rangle$ represents the dissipation parameters at the fourth-order level, $\left\langle\varepsilon^{2}\right\rangle$ provides indeed a better scaling than $\langle\varepsilon\rangle$ for quantities at that level. We plot the scaling exponents $\zeta_{[5]}$ in figure 5, as computed by

$$
\zeta_{[5]}=\frac{r}{S_{[5]}} \frac{\partial S_{[5]}}{\partial r},
$$

where again implicitly a power law for $S_{[5]}$ with constant prefactors and exponents is assumed. The dashed black horizontal line indicates the value $\zeta_{[5]}=1.56$. 


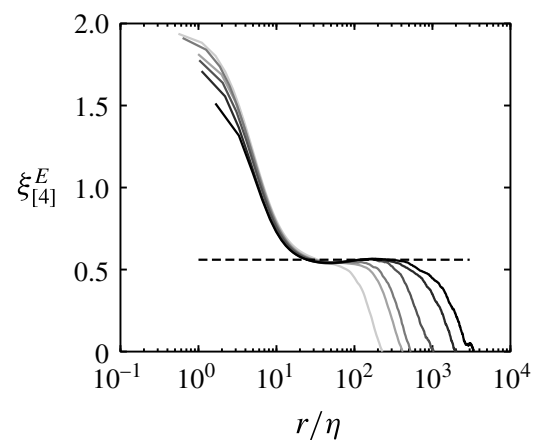

FIGURE 4. Scaling exponent $\xi_{[4]}^{E}$ for the cases $R 1-R 6$ with $R e_{\lambda}$ ranging from 119 to 754 (higher Reynolds numbers indicated by darker shading). The dashed black horizontal line indicates $\xi_{[4]}^{E}=0.56$.
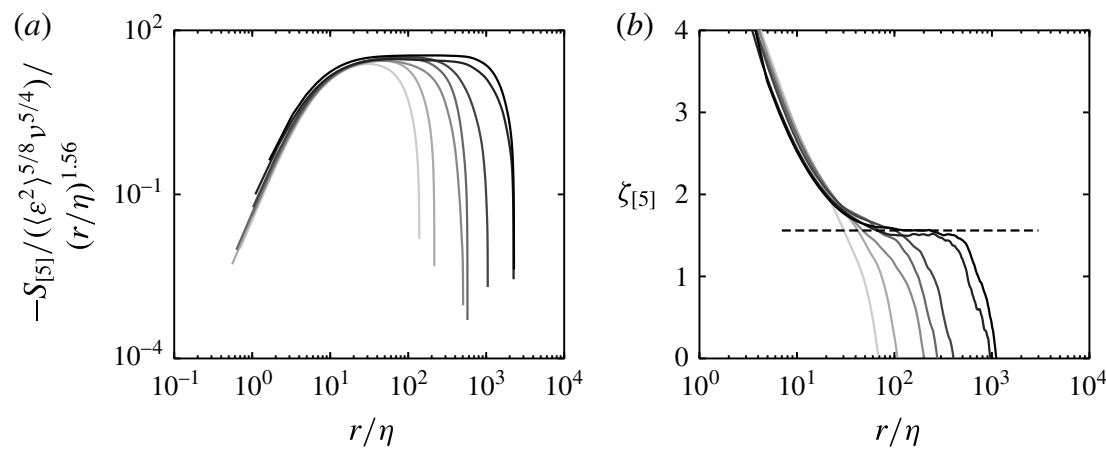

FIGURE 5. Compensated structure function $S_{[5]}$ in the inertial range for the cases $R 0$ $R 6$ with $R e_{\lambda}$ ranging from 88 to $754(a)$ and scaling exponent $\zeta_{[5]}(b)$. Higher Reynolds numbers are indicated by darker shading. The horizontal dashed black line in $(b)$ indicates $\zeta_{[5]}=1.56$.

In the next step, a relation between $\xi_{[4]}^{E}$ and the $\varepsilon^{2}$-term is needed. Therefore, we need to look at the transport equation of the dissipative source term $\left\langle E_{[4]}\right\rangle$ in more detail. However, under the assumption of stationarity, there is no term containing $\left\langle E_{[4]}\right\rangle$ in its transport equation when the inertial-range assumptions are invoked, as both the unsteady and the viscous terms are neglected. That is, information is lost by averaging, and, hence, additional assumptions and closures are needed. The situation is similar to the second-order structure function equations, where one finds in the inertial range a solution for the third-order structure functions (i.e. the 4/5 law), but all connections to the second order are lost. If one is interested in the solution of the second order in the inertial range, one has to make additional assumptions such as Kolmogorov's constant-skewness assumption.

That is, we would now need a relation between $\left\langle\Delta u_{1} E_{[4]}\right\rangle$ and $\left\langle E_{[4]}\right\rangle$, e.g. by employing a gradient flux ansatz. In the following, we rather use a slightly different approach. In analogy to K41 and K62 theory, one might postulate that fluctuations of the dissipation represent the relevant scaling parameter in the inertial range. Since the transport equation for $\left\langle E_{[4]}\right\rangle$ contains the $\varepsilon^{2}$-term, which represents dissipation fluctuations, it seems reasonable to scale the dissipative source term with $\varepsilon_{[4]}^{2}$ and $r$. 
It should be mentioned that the $r$-dependence of the $\varepsilon^{2}$-term is cancelled out by the $D$-term in the sum of the source terms. However, neither of the other terms combined with only $r$ is sufficient to provide a scaling of the dissipative source term without an additional parameter like $\delta_{[4]}^{E}$, and the same holds for the cancellations of $D_{[4]}+\varepsilon_{[4]}^{2}$ and $F_{[4]}+Q_{[4]}$. This indicates that some combination of the source terms in (3.1) is needed to scale the dissipative source term and ultimately $S_{[5]}$. Consequently, not only the dissipation fluctuations characterized by the $\varepsilon^{2}$-term are relevant, but also the other source terms in (3.1). However, the necessary combination of source terms cannot be determined by scaling arguments alone, because there are only two dimensions (m) and (s) and more than two scaling quantities. Furthermore, all terms are needed if the prefactor is also of interest.

In the following, we proceed to look only at the dissipation fluctuations, because they remain a quantity of high interest. We generalize the scaling of $\left\langle E_{[4]}\right\rangle$ with $\varepsilon_{[4]}^{2}$ and $r$ by adding a prefactor $\left(r / r^{*}\right)^{\delta_{[4]}^{E}}$, i.e. we use the ansatz

$$
\left\langle E_{[4]}\right\rangle=c_{[4]}^{E}\left(\varepsilon_{[4]}^{2}\right)^{5 / 6} r^{2 / 3+\delta_{[4]}^{E}} .
$$

Here, $r^{* \delta_{[4]}^{E}}$ is contained in $c_{[4]}^{E}$. Of course, this is an $a d$ hoc ansatz and only one of many possibilities. We do not want to claim that this is the best or only way to close the equations; rather, we use it for its simplicity. We use this closure for analytical purposes only, i.e. do not want to make predictions regarding higher orders, other data sets or flows. Because there are other source terms in (2.13) that contribute to the balance, one cannot expect that $\delta_{[4]}^{E}$ vanishes. Indeed, from our DNS, we find that $\delta_{[4]}^{E}=-0.09$. The numerical values of $\delta_{[4]}^{E}$ for $R 1-R 6$ are shown in figure $6(a)$, where

$$
\delta_{[4]}^{E}=r\left(\varepsilon_{[4]}^{2}\right)^{5 / 6}\left\langle E_{[4]}\right\rangle^{-1} \frac{\partial}{\partial r}\left(\left\langle E_{[4]}\right\rangle\left(\varepsilon_{[4]}^{2}\right)^{-5 / 6}\right)-\frac{2}{3}
$$

and the dashed horizontal black line corresponds to $\delta_{[4]}^{E}=-0.09$. This value is empirically determined and not claimed to be universal or to carry over to other flows. Moreover, its exact value is not important in the present context. We will now insert (4.11) into (4.7) and integrate. That is, we now examine the scaling of the dissipative source term $\left\langle E_{[4]}\right\rangle$ compared with the scaling of the $\varepsilon^{2}$-term, $\varepsilon_{[4]}^{2}$, which contains $\left\langle\varepsilon_{r}^{2}\right\rangle$. Using the ansatz (4.11), we find that this ratio should scale as $r^{2 / 3+\delta_{[4]}^{E}}$ in the inertial range, under the assumption that we may approximate the terms by power laws in the inertial range. The ratio $\left\langle E_{[4]}\right\rangle /\left(\varepsilon_{[4]}^{2} r^{\left.2 / 3+\delta_{[4]}^{E}\right)}\right.$ is shown in figure $6(b)$ for the cases $R 1-R 6$. We find a scaling range in the inertial range. In short, we have now replaced the dissipative source terms with $\varepsilon_{[4]}^{2} r^{2 / 3+\delta_{[4]}^{E}}$, which has the same $r$-scaling in the inertial range. If the $\varepsilon^{2}$-term has a power-law scaling in the inertial range, we can now integrate to obtain for the structure function trace

$$
S_{[5]} \sim r^{5 / 3+\delta_{[4]}^{E}}\left(\varepsilon_{[4]}^{2}\right)^{5 / 6} .
$$

This should be compared with the RSH, for which

$$
S_{[5]} \sim r^{5 / 3}\left\langle\varepsilon_{r}^{5 / 3}\right\rangle .
$$

That is, from the system of equations we would rather have a contribution from $\left\langle\varepsilon_{r}^{2}\right\rangle$, which is contained in $\varepsilon_{[4]}^{2}$, instead of $\left\langle\varepsilon_{r}^{5 / 3}\right\rangle$. As the RSH gives satisfactory results when compared with experimental data, the differences have to be contained in the 

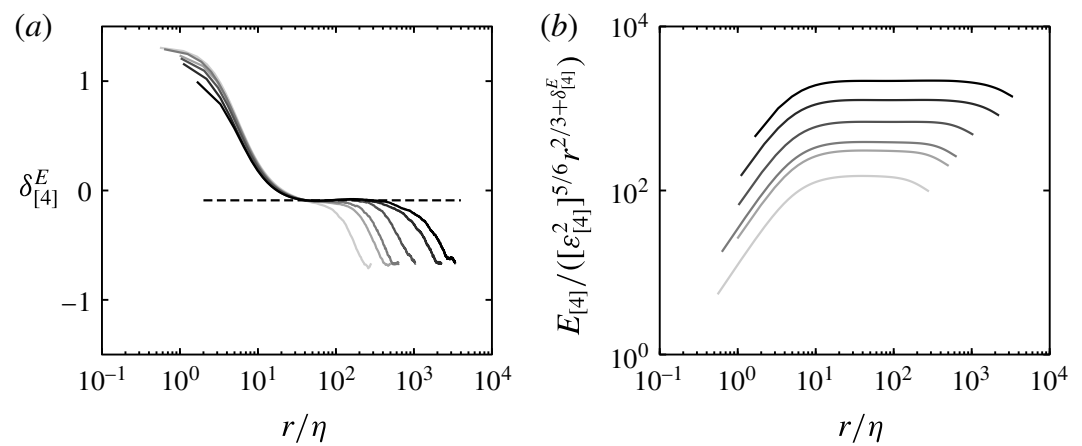

FIgURE 6. The term $\delta_{[4]}^{E}$ as evaluated by (4.12) for $R 1-R 6(a)$. Plot of the ratio $\left\langle E_{[4]}\right\rangle /\left(\varepsilon_{[4]}^{2} r^{2 / 3-\delta_{[4]}^{E}}\right)$ with $\delta_{[4]}^{E}=-0.09(b)$. Higher Reynolds numbers are indicated by darker shading.

parameter $\delta_{[4]}^{E}$, which contains empirically the influence of the other source terms in (3.1) on the dissipative source term $\left\langle E_{[4]}\right\rangle$. It should be noted that (4.14) implies $\left\langle E_{[4]}\right\rangle \sim r^{2 / 3}\left\langle\varepsilon_{r}^{5 / 3}\right\rangle$, if a power law for $\varepsilon_{r}$ can be assumed. However, there is no term containing $\left\langle\varepsilon_{r}^{5 / 3}\right\rangle$ in either the fourth- or the fifth-order equations.

We may conclude that the RSH assumption of using $\varepsilon_{r}$ instead of the K41 ansatz using $\langle\varepsilon\rangle$ is compatible with the results of our approach here, in the sense that $\varepsilon_{r}$ appears in the $\varepsilon^{2}$-term. However, we find in the system of equations a contribution to the fifth-order structure functions by $\left\langle\varepsilon_{r}^{2}\right\rangle$; i.e. the connection to $\left\langle\varepsilon_{r}^{n / 3}\right\rangle$ is still missing. We expect similar results at higher orders, which should then be related to higher moments of $\varepsilon_{r}$. However, the pressure source terms also might be important at higher orders, which one would then need to close as well. Furthermore, we find that the $r$-scaling of the $\varepsilon^{2}$-term is cancelled out by the $D$-term in the full system of equations. We are left with the fact that while we have found all higher moments of $\varepsilon_{r}$ in the dissipative source term equations and further equations derived therefrom, we cannot say why $n$ th-order structure functions should be determined by $\left\langle\varepsilon_{r}^{n / 3}\right\rangle$, i.e. by the RSH or other similar theories. While the numerical values obtained in either way agree fairly well (at least for the fourth order we examined here), such a connection cannot be obtained from the Navier-Stokes equations without additional empirical closures.

The analysis can be carried to higher orders. It can easily be shown that the third successive equation for dissipation source terms in the trace of the eighth-order structure function equations contains a term $\left\langle\left(\varepsilon_{i i}+\varepsilon_{i i}^{\prime}\right)\left(\varepsilon_{j j}+\varepsilon_{j j}^{\prime}\right)\left(\varepsilon_{k k}+\varepsilon_{k k}^{\prime}\right)\left(\varepsilon_{l l}+\varepsilon_{l l}^{\prime}\right)\right\rangle$, which generates a dissipation parameter $\left\langle\varepsilon^{4}\right\rangle$. As one continues further, one will find all moments of the dissipation distribution function in the system of averaged equations. On the basis of the equations at the sixth-order structure function level, for instance, one could perform similar integrations to those for the fourth-order level. For instance, in the archive material, we have derived an equation for the dissipation source term $\left\langle E_{6,0}\right\rangle=15\left\langle\left(\Delta u_{1}\right)^{4}\left(\varepsilon_{11}+\varepsilon_{11}^{\prime}\right)\right\rangle$ which appears in the sixth-order longitudinal structure function equation. In the equation for $\left\langle E_{6,0}\right\rangle$, a source term $\left\langle F_{6,0}\right\rangle=15\left\langle\left(\Delta u_{1}\right)^{2}\left(\varepsilon_{11}+\varepsilon_{11}^{\prime}\right)^{2}\right\rangle$ appears. In the equation for $\left\langle F_{6,0}\right\rangle$, the term $15\left\langle\left(\varepsilon_{11}+\varepsilon_{11}^{\prime}\right)^{3}\right\rangle$ appears, which generates the new dissipation parameter $\left\langle\varepsilon_{11}^{3}\right\rangle$, and combined with the mixed and transverse equations, one finds an $\varepsilon_{[6]}^{3}$-term which contains $\left\langle\left(\varepsilon+\varepsilon^{\prime}\right)^{3}\right\rangle$, i.e. one would expect to find a contribution by $\left\langle\varepsilon_{r}^{3}\right\rangle$ in the sixth-order equations. In order to calculate seventh-order inertial-range scaling 
coefficients from the sixth-order structure function equations and their dissipation source terms, the trace of the structure function equations and two successive source term equations would have to be integrated. An ansatz similar to (4.11) would provide at leading order the exponent $7 / 3$. The deviation from this K41 scaling exponent would then involve more empirical terms. Similar arguments will hold for all other uneven-order scaling exponents. Because there are no dissipation parameters in the uneven-order source term equations, even-order scaling exponents cannot be determined in this way.

\section{Conclusion}

In addition to $v$ and $\langle\varepsilon\rangle$, all higher-order moments of the dissipation distribution function appear as dissipation parameters in the extended system of two-point equations for small-scale turbulence. The effect of the higher-order dissipation parameters on the solutions of these equations is demonstrated for the trace of the fourth-order structure function equations, which are invariant, independent of the coordinate system. The procedure can also be carried over to the individual structure function equations at higher even orders; for example, in the sixth-order equations, the third-order dissipation parameters $\left\langle\left(\varepsilon_{11}+\varepsilon_{11}^{\prime}\right)^{3}\right\rangle$ etc. are found. The analysis uses exact equations, but because of the inherent closure problem needs to apply empirical closure assumptions between some of the terms. The conclusions are twofold. (1) The higher-order dissipation parameters determine the solutions of the even-order structure functions in the viscous range. (2) The trace of the dissipation parameters in the system of equations contains the moments of $\varepsilon_{r}$. However, the relation of the specific assumption $\left\langle\varepsilon_{r}^{n / 3}\right\rangle$ for the $n$ th-order structure functions in the inertial range to the dissipation parameters derived from the Navier-Stokes equations is missing.

\section{Acknowledgements}

This work was supported by the Deutsche Forschungsgemeinschaft through the grant Pe 241/38-2. The authors gratefully acknowledge the computing time granted by the JARA-HPC-Vergabegremium provided on the JARA-HPC Partition part of the supercomputer JUQUEEN at the Forschungszentrum Jülich.

\section{Appendix A. Direct numerical simulation (DNS) in a cubic box for Taylor-based Reynolds numbers up to 529}

We solve the three-dimensional Navier-Stokes equations for incompressible fluids in rotational form,

$$
\frac{\partial \boldsymbol{u}}{\partial t}+\boldsymbol{\omega} \times \boldsymbol{u}=-\nabla\left(p+\frac{1}{2} \boldsymbol{u}^{2}\right)+v \nabla^{2} \boldsymbol{u}+\boldsymbol{f},
$$

with the continuity equation

$$
\nabla \cdot \boldsymbol{u}=\mathbf{0}
$$

where $\boldsymbol{\omega}=\boldsymbol{\nabla} \times \boldsymbol{u}$ is the vorticity, $p$ is the ratio of pressure and density, and $v$ is the kinematic viscosity; $\boldsymbol{f}$ is a forcing term which acts on large scales only.

Equation (A 1) is solved by a pseudo-spectral approach in Fourier space, cf. Rogallo (1981) and Canuto et al. (1988). Temporal integration is carried out by an explicit second-order Adams-Bashforth method, cf. Butcher (2004). The integration domain is 


\begin{tabular}{|c|c|c|c|c|c|c|c|}
\hline & $R 0$ & $R 1$ & $R 2$ & $R 3$ & $R 4$ & $R 5$ & $R 6$ \\
\hline$N$ & 512 & 1024 & 1024 & 2048 & 2048 & 4096 & 4096 \\
\hline$R e_{\lambda}$ & 88 & 119 & 184 & 215 & 331 & 529 & 754 \\
\hline$v$ & 0.01 & 0.0055 & 0.0025 & 0.0019 & 0.0010 & 0.00048 & 0.00027 \\
\hline$\langle k\rangle$ & 11.15 & 11.38 & 11.42 & 12.70 & 14.35 & 23.95 & 24.42 \\
\hline$\langle\varepsilon\rangle$ & 10.78 & 11.04 & 10.30 & 11.87 & 12.55 & 28.51 & 26.54 \\
\hline$\eta$ & 0.0175 & 0.0111 & 0.0062 & 0.0049 & 0.0030 & 0.0014 & 0.00093 \\
\hline$\tau$ & 1.03 & 1.03 & 1.11 & 1.07 & 1.14 & 0.84 & 0.92 \\
\hline$t_{a v g} / \tau$ & 100 & 30 & 30 & 10 & 10 & 2 & 3 \\
\hline$M$ & 189 & 62 & 61 & 10 & 10 & 6 & 6 \\
\hline$\Delta t$ & $1.4 \times 10^{-4}$ & $8.0 \times 10^{-5}$ & $8.0 \times 10^{-5}$ & $2.85 \times 10^{-5}$ & $2.75 \times 10^{-5}$ & $1.01 \times 10^{-5}$ & $1.01 \times 10^{-5}$ \\
\hline$\kappa_{\max } \eta$ & 3.93 & 4.99 & 2.93 & 4.41 & 2.53 & 2.95 & 1.76 \\
\hline
\end{tabular}

a triply periodic cube with length $2 \pi$. An external stochastic forcing, cf. Eswaran \& Pope (1988), is applied to the velocity field to maintain a statistically steady state. The simulations have been carried out with an in-house hybrid MPI/OpenMP parallelized simulation code which efficiently utilizes the BlueGene/Q architecture.

Characteristic parameters of the DNS are listed in table 1 . Here, $N$ denotes the number of grid points in one direction, $R e_{\lambda}$ the Taylor-based Reynolds number, $\langle k\rangle$ the mean turbulent kinetic energy, $\langle\varepsilon\rangle$ the mean energy dissipation rate, $\tau=\langle k\rangle /\langle\varepsilon\rangle$ the integral time scale, $v$ the kinematic viscosity, $\eta=\left(v^{3} /\langle\varepsilon\rangle\right)^{1 / 4}$ the Kolmogorov length scale and $\tau$ the integral time scale, whereas $t_{\text {avg }}$ is the average time up to which the simulations have been performed; $M$ is the number of independent data sets over which averages have been taken and $\Delta t$ is the time step. Following Ishihara et al. (2007), a resolution $k_{\max } \eta>1.7$ is maintained in order to accurately compute higher-order statistics.

\section{Appendix B. Longitudinal, transverse and mixed fourth-order structure function and dissipative source term equations}

Here, the longitudinal, mixed and transverse fourth-order structure function equations are briefly presented. A detailed derivation can be found in Hill (2001). In their homogeneous isotropic form, the structure function equations read as follows.

Longitudinal:

$$
\begin{aligned}
& \frac{\partial S_{4,0}}{\partial t}+\frac{\partial S_{5,0}}{\partial r}+\frac{2}{r} S_{5,0}-\frac{8}{r} S_{3,2} \\
& \quad=-\left\langle T_{4,0}\right\rangle-\left\langle E_{4,0}\right\rangle+2 v\left[\frac{\partial^{2} S_{4,0}}{\partial r^{2}}+\frac{2}{r} \frac{\partial S_{4,0}}{\partial r}-\frac{8}{r^{2}} S_{4,0}+\frac{24}{r^{2}} S_{2,2}\right]
\end{aligned}
$$

Mixed:

$$
\begin{aligned}
& \frac{\partial S_{2,2}}{\partial t}+\frac{\partial S_{3,2}}{\partial r}+\frac{4}{r} S_{3,2}-\frac{8}{3 r} S_{1,4} \\
& \quad=-\left\langle T_{2,2}\right\rangle-\left\langle E_{2,2}\right\rangle+2 v\left[\frac{2}{r^{2}} S_{4,0}+\frac{\partial^{2} S_{2,2}}{\partial r^{2}}+\frac{2}{r} \frac{\partial S_{2,2}}{\partial r}-\frac{14}{r^{2}} S_{2,2}+\frac{8}{3 r^{2}} S_{0,4}\right]
\end{aligned}
$$


Transverse:

$$
\frac{\partial S_{0,4}}{\partial t}+\frac{\partial S_{1,4}}{\partial r}+\frac{6}{r} S_{1,4}=-\left\langle T_{0,4}\right\rangle-\left\langle E_{0,4}\right\rangle+2 v\left[\frac{12}{r^{2}} S_{2,2}+\frac{\partial^{2} S_{0,4}}{\partial r^{2}}+\frac{2}{r} \frac{\partial S_{0,4}}{\partial r}-\frac{4}{r^{2}} S_{0,4}\right] .
$$

The coefficients in the viscous terms differ from those given by Hill (2001). Our derivation may be found in the archive material. In agreement with the present formulae, http://arxiv.org/abs/physics/0102055 (derivation for Hill (2001)) now contains corrected viscous-term formulae, matrix algorithm and table of formulae. The pressure source terms $\left\langle T_{4,0}\right\rangle,\left\langle T_{2,2}\right\rangle$ and $\left\langle T_{0,4}\right\rangle$ are defined as

$$
\begin{gathered}
\left\langle T_{4,0}\right\rangle=\left\langle 4\left(\Delta u_{1}\right)^{3} \Delta P_{1}\right\rangle, \\
\left\langle T_{2,2}\right\rangle=\left\langle 2\left(\Delta u_{2}\right)^{2} \Delta u_{1} \Delta P_{1}+2\left(\Delta u_{1}\right)^{2} \Delta u_{2} \Delta P_{2}\right\rangle, \\
\left\langle T_{0,4}\right\rangle=\left\langle 4\left(\Delta u_{2}\right)^{3} \Delta P_{2}\right\rangle,
\end{gathered}
$$

where $\Delta P_{i}=\left(\partial p / \partial x_{i}-\partial p^{\prime} / \partial x_{i}^{\prime}\right)$ is the difference of pressure gradients at the two points. The dissipation source terms $\left\langle E_{4,0}\right\rangle,\left\langle E_{2,2}\right\rangle$ and $\left\langle E_{0,4}\right\rangle$ are defined as

$$
\begin{gathered}
\left\langle E_{4,0}\right\rangle=\left\langle 6\left(\Delta u_{1}\right)^{2}\left(\varepsilon_{11}+\varepsilon_{11}^{\prime}\right)\right\rangle, \\
\left\langle E_{2,2}\right\rangle=\left\langle\left(\Delta u_{2}\right)^{2}\left(\varepsilon_{11}+\varepsilon_{11}^{\prime}\right)+4 \Delta u_{1} \Delta u_{2}\left(\varepsilon_{12}+\varepsilon_{12}^{\prime}\right)+\left(\Delta u_{1}\right)^{2}\left(\varepsilon_{22}+\varepsilon_{22}^{\prime}\right)\right\rangle, \\
\left\langle E_{0,4}\right\rangle=\left\langle 6\left(\Delta u_{2}\right)^{2}\left(\varepsilon_{22}+\varepsilon_{22}^{\prime}\right)\right\rangle
\end{gathered}
$$

respectively, with $\varepsilon_{i j}=2 v\left(\partial u_{i} / \partial x_{k}\right)\left(\partial u_{j} / \partial x_{k}\right)$.

Noticeably enough, we have three equations for three unknown structure functions in the viscous range as well as under the inertial-range assumptions. This implies that we can then integrate the equations starting with (B 3), inserting the solution into (B 2), integrating this equation, and then finally solve (B 1), if the source terms are known. This characteristic is found for all even orders, but not for odd orders. Indeed, one can obtain Kolmogorov's 4/5 and 4/15 laws by integrating the second-order equations in the same manner, cf. Hill (1997).

The individual equations for the fourth-order dissipative source terms are given in the archive material. The transport equation for $\left\langle E_{4,0}\right\rangle$ reads

$$
\begin{aligned}
& \frac{\partial\left\langle\Delta u_{1} E_{4,0}\right\rangle}{\partial r}+\frac{2}{r}\left\langle\Delta u_{1} E_{4,0}\right\rangle-\frac{8}{r}\left\langle\Delta u_{2} E_{3,1}\right\rangle \\
& =2 v\left[\frac{\partial^{2}\left\langle E_{4,0}\right\rangle}{\partial r^{2}}+\frac{2}{r} \frac{\partial\left\langle E_{4,0}\right\rangle}{\partial r}-\frac{8}{r^{2}}\left\langle E_{4,0}\right\rangle+\frac{24}{r^{2}}\left\langle E_{2,2}\right\rangle\right]-\sum P_{4,0}^{E},
\end{aligned}
$$

where the sum of source terms is

$$
\begin{aligned}
\sum P_{4,0}^{E}= & 24 v\left\langle\left(\Delta u_{1}\right)^{2}\left(A_{11}+A_{11}^{\prime}\right)\right\rangle+12 v\left\langle\left(\Delta u_{1}\right)^{2}\left(\chi_{11}+\chi_{11}^{\prime}\right)\right\rangle \\
& +12\left\langle\Delta u_{1} \Delta P_{1}\left(\varepsilon_{11}+\varepsilon_{11}^{\prime}\right)\right\rangle+24 v\left\langle\Delta u_{1} \Delta u_{1}\left(P_{11}+P_{11}^{\prime}\right)\right\rangle \\
& +12 v\left\langle\left(\frac{\partial\left(\Delta u_{1}\right)^{2}}{\partial x_{n}} \frac{\partial \varepsilon_{11}}{\partial x_{n}}+\frac{\partial\left(\Delta u_{1}\right)^{2}}{\partial x_{n}^{\prime}} \frac{\varepsilon_{11}^{\prime}}{\partial x_{n}^{\prime}}\right)\right\rangle+6\left\langle\left(\varepsilon_{11}+\varepsilon_{11}^{\prime}\right)^{2}\right\rangle .
\end{aligned}
$$

For the mixed dissipative source term $\left\langle E_{2,2}\right\rangle$ one obtains the transport equation

$$
\begin{aligned}
& \frac{\partial\left\langle\Delta u_{1} E_{2,2}\right\rangle}{\partial r}+\frac{2}{r}\left\langle\Delta u_{1} E_{2,2}\right\rangle+\frac{2}{r}\left\langle\Delta u_{2} E_{3,1}\right\rangle-\frac{8}{3 r}\left\langle\Delta u_{2} E_{1,3}\right\rangle \\
& =2 v\left[\frac{\partial^{2}\left\langle E_{2,2}\right\rangle}{\partial r^{2}}+\frac{2}{r} \frac{\partial\left\langle E_{2,2}\right\rangle}{\partial r}-\frac{14}{r^{2}}\left\langle E_{2,2}\right\rangle+\frac{2}{r^{2}}\left\langle E_{4,0}\right\rangle+\frac{8}{3 r^{2}}\left\langle E_{0,4}\right\rangle\right]-\sum P_{2,2}^{E},
\end{aligned}
$$


where the sum of source terms is

$$
\begin{aligned}
\sum P_{2,2}^{E}= & 2 v\left\langle 2\left(\Delta u_{1}\right)^{2}\left(A_{22}+A_{22}^{\prime}\right)+4 \Delta u_{1} \Delta u_{2}\left(A_{12}+A_{12}^{\prime}+A_{21}+A_{21}^{\prime}\right)\right. \\
& \left.+2\left(\Delta u_{2}\right)^{2}\left(A_{11}+A_{11}^{\prime}\right)\right\rangle+2 v\left\langle\left(\Delta u_{1}\right)^{2}\left(\chi_{22}+\chi_{22}^{\prime}\right)+2 \Delta u_{1} \Delta u_{2}\left(\chi_{12}+\chi_{12}^{\prime}\right)\right. \\
& \left.+\left(\Delta u_{2}\right)^{2}\left(\chi_{11}+\chi_{11}^{\prime}\right)\right\rangle+2\left\langle\Delta u_{1} \Delta P_{1}\left(\varepsilon_{22}+\varepsilon_{22}^{\prime}\right)\right. \\
& \left.+2\left(\Delta u_{1} \Delta P_{2}+\Delta u_{2} \Delta P_{1}\right)\left(\varepsilon_{12}+\varepsilon_{12}^{\prime}\right)+\Delta u_{2} \Delta P_{2}\left(\varepsilon_{11}+\varepsilon_{11}^{\prime}\right)\right\rangle \\
& +2 v\left\langle 2\left(\Delta u_{1}\right)^{2}\left(P_{22}+P_{22}^{\prime}\right)+4 \Delta u_{1} \Delta u_{2}\left(P_{12}+P_{12}^{\prime}+P_{21}+P_{21}^{\prime}\right)\right. \\
& \left.+2\left(\Delta u_{2}\right)^{2}\left(P_{11}+P_{11}^{\prime}\right)\right\rangle+2 v\left\langle\left(\frac{\partial\left(\Delta u_{1}\right)^{2}}{\partial x_{n}} \frac{\partial \varepsilon_{22}}{\partial x_{n}}+4 \frac{\partial \Delta u_{1} \Delta u_{2}}{\partial x_{n}} \frac{\partial \varepsilon_{12}}{\partial x_{n}}\right.\right. \\
& \left.\left.+\frac{\partial\left(\Delta u_{2}\right)^{2}}{\partial x_{n}} \frac{\partial \varepsilon_{11}}{\partial x_{n}}+\frac{\partial\left(\Delta u_{1}\right)^{2}}{\partial x_{n}^{\prime}} \frac{\varepsilon_{22}^{\prime}}{\partial x_{n}^{\prime}}+\frac{\partial \Delta u_{1} \Delta u_{2}}{\partial x_{n}^{\prime}} \frac{\varepsilon_{12}^{\prime}}{\partial x_{n}^{\prime}}+\frac{\partial\left(\Delta u_{2}\right)^{2}}{\partial x_{n}^{\prime}} \frac{\varepsilon_{11}^{\prime}}{\partial x_{n}^{\prime}}\right)\right\rangle \\
& +2\left\langle\left(\varepsilon_{11}+\varepsilon_{11}^{\prime}\right)\left(\varepsilon_{22}+\varepsilon_{22}^{\prime}\right)+\left(\varepsilon_{12}+\varepsilon_{12}^{\prime}\right)\left(\varepsilon_{12}+\varepsilon_{12}^{\prime}\right)\right\rangle .
\end{aligned}
$$

The transport equation for the transverse dissipation source term $\left\langle E_{0,4}\right\rangle$ is

$$
\begin{aligned}
& \frac{\partial\left\langle\Delta u_{1} E_{0,4}\right\rangle}{\partial r}+\frac{2}{r}\left\langle\Delta u_{1} E_{0,4}\right\rangle+\frac{4}{r}\left\langle\Delta u_{2} E_{1,3}\right\rangle \\
& =2 v\left[\frac{\partial^{2}\left\langle E_{0,4}\right\rangle}{\partial r^{2}}+\frac{2}{r} \frac{\partial\left\langle E_{0,4}\right\rangle}{\partial r}-\frac{4}{r}\left\langle E_{0,4}\right\rangle+\frac{12}{r^{2}}\left\langle E_{2,2}\right\rangle\right]-\sum P_{0.4}^{E}
\end{aligned}
$$

where the sum of the source term is

$$
\begin{aligned}
\sum P_{0,4}^{E}= & 24 v\left\langle\left(\Delta u_{2}\right)^{2}\left(A_{2,2}+A_{2,2}^{\prime}\right)\right\rangle+12 v\left\langle\left(\Delta u_{2}\right)^{2}\left(\chi_{22}+\chi_{22}^{\prime}\right)\right\rangle \\
& +12\left\langle\Delta u_{2} \Delta P_{2}\left(\varepsilon_{22}+\varepsilon_{22}^{\prime}\right)\right\rangle+24 v\left\langle\Delta u_{2}^{2}\left(P_{22}+P_{22}^{\prime}\right)\right\rangle \\
& +12 v\left\langle\frac{\partial \Delta u_{2}^{2}}{\partial x_{n}} \frac{\partial \varepsilon_{22}}{\partial x_{n}}+\frac{\partial \Delta u_{2}^{2}}{\partial x_{n}^{\prime}} \frac{\partial \varepsilon_{22}^{\prime}}{\partial x_{n}^{\prime}}\right\rangle+6\left\langle\left(\varepsilon_{22}+\varepsilon_{22}^{\prime}\right)^{2}\right\rangle
\end{aligned}
$$

That is, we find the second-order dissipation parameters $\left\langle\left(\varepsilon_{i j}+\varepsilon_{i j}^{\prime}\right)\left(\varepsilon_{k l}+\varepsilon_{k l}^{\prime}\right)\right\rangle$ in the fourth-order dissipative source term equations.

\section{Appendix C. Relations between fourth-order structure functions and second-order dissipation parameters in the viscous range}

In this section we will briefly discuss the individual fourth-order structure functions in the viscous range.

In the viscous range, we need to consider only the balance between the viscous terms and the dissipation source terms in the fourth-order longitudinal, mixed and transverse structure functions, cf. figure 1:

$$
\begin{gathered}
2 v\left[\frac{\partial^{2} S_{4,0}}{\partial r^{2}}+\frac{2}{r} \frac{\partial S_{4,0}}{\partial r}-\frac{8}{r^{2}} S_{4,0}+\frac{24}{r^{2}} S_{2,2}\right]=\left\langle E_{4,0}\right\rangle, \\
2 v\left[\frac{2}{r^{2}} S_{4,0}+\frac{\partial^{2} S_{2,2}}{\partial r^{2}}+\frac{2}{r} \frac{\partial S_{2,2}}{\partial r}-\frac{14}{r^{2}} S_{2,2}+\frac{8}{3 r^{2}} S_{0,4}\right]=\left\langle E_{2,2}\right\rangle, \\
2 v\left[\frac{12}{r^{2}} S_{2,2}+\frac{\partial^{2} S_{0,4}}{\partial r^{2}}+\frac{2}{r} \frac{\partial S_{0,4}}{\partial r}-\frac{8}{r^{2}} S_{0,4}\right]=\left\langle E_{0,4}\right\rangle .
\end{gathered}
$$


In the limit $r \rightarrow 0$, the viscous terms balance to leading order. In the dissipative source term equations, the viscous terms balance the $\varepsilon^{2}$-terms, resulting in

$$
\begin{gathered}
2 v\left[\frac{\partial^{2}\left\langle E_{4,0}\right\rangle}{\partial r^{2}}+\frac{2}{r} \frac{\partial\left\langle E_{4,0}\right\rangle}{\partial r}-\frac{8}{r^{2}}\left\langle E_{4,0}\right\rangle+\frac{24}{r^{2}}\left\langle E_{2,2}\right\rangle\right]=6\left\langle\left(\varepsilon_{11}+\varepsilon_{11}^{\prime}\right)^{2}\right\rangle, \\
2 v\left[\frac{2}{r^{2}}\left\langle E_{4,0}\right\rangle+\frac{\partial^{2}\left\langle E_{2,2}\right\rangle}{\partial r^{2}}+\frac{2}{r} \frac{\partial\left\langle E_{2,2}\right\rangle}{\partial r}-\frac{14}{r^{2}}\left\langle E_{2,2}\right\rangle+\frac{8}{3 r^{2}}\left\langle E_{0,4}\right\rangle\right] \\
=2\left\langle\left(\varepsilon_{11}+\varepsilon_{11}^{\prime}\right)\left(\varepsilon_{22}+\varepsilon_{22}^{\prime}\right)\right\rangle+4\left\langle\left(\varepsilon_{12}+\varepsilon_{12}^{\prime}\right)^{2}\right\rangle, \\
2 v\left[\frac{12}{r^{2}}\left\langle E_{2,2}\right\rangle+\frac{\partial^{2}\left\langle E_{0,4}\right\rangle}{\partial r^{2}}+\frac{2}{r} \frac{\partial\left\langle E_{0,4}\right\rangle}{\partial r}-\frac{8}{r^{2}}\left\langle E_{0,4}\right\rangle\right]=6\left\langle\left(\varepsilon_{22}+\varepsilon_{22}^{\prime}\right)^{2}\right\rangle .
\end{gathered}
$$

Expanding the structure functions as in (3.4)-(3.6) and the dissipation source terms as

$$
\begin{aligned}
E_{4,0}= & \beta_{4,0}^{0} r^{2}+\cdots=12\left\langle\left(\frac{\partial u_{1}}{\partial x_{1}}\right)^{2} \varepsilon_{11}\right\rangle r^{2} \ldots, \\
E_{2,2}=\beta_{2,2}^{0} r^{2}+\cdots= & \left(2\left\langle\left(\frac{\partial u_{1}}{\partial x_{1}}\right)^{2} \varepsilon_{11}\right\rangle\right. \\
& \left.+8\left\langle\left(\frac{\partial u_{1}}{\partial x_{1}}\right)\left(\frac{\partial u_{2}}{\partial x_{1}}\right) \varepsilon_{12}\right\rangle+2\left\langle\left(\frac{\partial u_{1}}{\partial x_{1}}\right)^{2} \varepsilon_{22}\right\rangle\right) r^{2} \ldots, \\
E_{0,4} & =\beta_{4,0}^{0} r^{2}+\cdots=12\left\langle\left(\frac{\partial u_{2}}{\partial x_{1}}\right)^{2}\right\rangle r^{2} \ldots,
\end{aligned}
$$

we obtain to leading order the relations for $F_{1}, F_{2}$ and $F_{3}$ :

$$
\begin{gathered}
12 F_{1}+24 F_{2}=\frac{\beta_{4,0}^{0}}{2 v}, \\
2 F_{1}+6 F_{2}+\frac{8}{3} F_{3}=\frac{\beta_{2,2}^{0}}{2 v}, \\
12 F_{2}+16 F_{3}=\frac{\beta_{0,4}^{0}}{2 v} .
\end{gathered}
$$

Gauss elimination leads to a singular system and thereby to the compatibility condition

$$
\beta_{4,0}^{0}+\beta_{0,4}^{0}=6 \beta_{2,2}^{0} \text {. }
$$

By inserting (C 10)-(C 12) into the equations for the fourth-order dissipation source terms, we obtain to leading order the relations

$$
\begin{gathered}
-2 \beta_{4,0}^{0}+24 \beta_{2,2}^{0}=24 \frac{\left\langle\varepsilon_{11}^{2}\right\rangle}{2 v}, \\
2 \beta_{4,0}^{0}-8 \beta_{2,2}^{0}+\frac{8}{3} \beta_{0,4}^{0}=\frac{8\left\langle\varepsilon_{11} \varepsilon_{22}\right\rangle+16\left\langle\varepsilon_{12}^{2}\right\rangle}{2 v}, \\
12 \beta_{2,2}^{0}+2 \beta_{0,4}^{0}=24 \frac{\left\langle\varepsilon_{22}^{2}\right\rangle}{2 v} .
\end{gathered}
$$




$\begin{array}{lccccccc}R e_{\lambda} & 88 & 119 & 184 & 215 & 331 & 529 & 680 \text { (Ishihara et al.) } \\ I_{2} / I_{1} & 1.6726 & 1.6951 & 1.40015 & 1.7247 & 1.7347 & 1.7443 & 1.75 \\ I_{3} / I_{1} & 0.2132 & 0.2181 & 0.2236 & 0.2267 & 0.2294 & 0.2341 & 0.24 \\ I_{4} / I_{1} & 6.6402 & 7.0349 & 7.3893 & 7.5573 & 7.7334 & 7.9494 & 8.0\end{array}$

TABLE 2. Ratios of invariants of the fourth-order velocity derivative tensor.

Gauss elimination leads again to a singular system and a compatibility condition relating the dissipation parameters,

$$
3\left\langle\varepsilon_{11}^{2}\right\rangle+\left\langle\varepsilon_{11} \varepsilon_{22}\right\rangle+2\left\langle\varepsilon_{12}^{2}\right\rangle=4\left\langle\varepsilon_{22}^{2}\right\rangle .
$$

Since, due to isotropy, $\left\langle\varepsilon_{11}^{2}\right\rangle=\left\langle\varepsilon_{22}^{2}\right\rangle$ and (C 17) may be written as

$$
\left\langle\varepsilon_{11}^{2}\right\rangle-\left\langle\varepsilon_{11} \varepsilon_{22}\right\rangle-2\left\langle\varepsilon_{12}^{2}\right\rangle=0,
$$

by using (C 10) and (C 11) in (C 12), one obtains a relation between $F_{1}, F_{2}, F_{3}$ and $\left\langle\varepsilon_{11}^{2}\right\rangle$ as

$$
4 F_{1}+16 F_{2}+\frac{32}{3} F_{3}=\frac{\left\langle\varepsilon_{11}^{2}\right\rangle}{v^{2}} .
$$

Using (3.8), all second-order dissipation parameters may be expressed in terms of Siggia's invariants,

$$
\frac{\left\langle\varepsilon_{11}^{2}\right\rangle}{v^{2}}=\frac{\left\langle\varepsilon_{22}^{2}\right\rangle}{v^{2}}=\frac{\left\langle\varepsilon_{11} \varepsilon_{22}\right\rangle+2\left\langle\varepsilon_{12}^{2}\right\rangle}{v^{2}}=\frac{8}{15} I_{1}+\frac{16}{15} I_{2}-\frac{16}{15} I_{3}+\frac{2}{15} I_{4} .
$$

As noted by Siggia (1981), the ratios $I_{2} / I_{1}, I_{3} / I_{1}$ and $I_{4} / I_{1}$ should be Reynolds-numberindependent for large Reynolds numbers. This is shown to be approximately true for the Reynolds numbers of our DNS calculations as shown in table 2, where the ratios are also compared with the values obtained by Ishihara et al. (2007) calculated at $R e_{\lambda}=680$. Taking the numbers of Ishihara et al. (2007) for these ratios, we obtain with $I_{1}=\left\langle\varepsilon^{2}\right\rangle /\left(4 v^{2}\right)$

$$
\left\langle\varepsilon_{11}^{2}\right\rangle=\left\langle\varepsilon_{22}^{2}\right\rangle=\left\langle\varepsilon_{11} \varepsilon_{22}\right\rangle+2\left\langle\varepsilon_{12}^{2}\right\rangle \approx 0.8\left\langle\varepsilon^{2}\right\rangle .
$$

All second-order dissipation parameters should therefore scale with the Reynolds number in the same way as $\left\langle\varepsilon^{2}\right\rangle$.

\section{REFERENCES}

Benzi, R., Biferale, L. \& Toschi, F. 1998 Multiscale velocity correlations in turbulence. Phys. Rev. Lett. 80 (15), 3244-3247.

Butcher, J. C. 2004 Numerical Methods for Ordinary Differential Equations. Wiley.

Canuto, C., Hussaini, M. Y., Quarteroni, A. \& Zang, T. A. 1988 Spectral Methods in Fluid Dynamics. Springer.

Dubrulle, B. 1994 Intermittency in fully developed turbulence: log-Poisson statistics and generalized scale-covariance. Phys. Rev. Lett. 73, 959-962.

EsWARAN, V. \& POPE, S. B. 1988 An examination of forcing in direct numerical simulations of turbulence. Comput. Fluids 16, 257-278. 
Fairhall, A. L., Dhruva, B., L'vov, V., Procaccia, I. \& Sreenivasan, K. R. 1997 Fusion rules in Navier-Stokes turbulence: first experimental tests. Phys. Rev. Lett. 79 (17), 3174-3177.

Falkovich, G., Fouxon, I. \& Oz, Y. 2010 New relations for correlation functions in Navier-Stokes turbulence. J. Fluid Mech. 644, 465-472.

FRISCH, U. 1995 Turbulence: The Legacy of A.N. Kolmogorov. Cambridge University Press.

Gotoh, T. \& Nakano, T. 2003 Role of pressure in turbulence. J. Stat. Phys. 113 (5-6), 855-874.

Hill, R. J. 1997 Applicability of Kolmogorov's and Monin's equations of turbulence. J. Fluid Mech. 353, 67-81.

HiLl, R. J. 2001 Equations relating structure functions of all orders. J. Fluid Mech. 434, 379-388.

HILl, R. J. 2002 Exact second-order structure-function relationships. J. Fluid Mech. 468, 317-326.

Hill, R. J. \& Boratav, O. N. 2001 Next-order structure function equations. Phys. Fluids 13, 276-283.

Ishihara, T., Kaneda, Y., Yokokawa, M. \& Uno, K. A. 2007 Small scale statistics in highresolution direct numerical simualtion of turbulence: Reynolds number dependence in one-point velocity gradient statistics. J. Fluid Mech. 592, 335-366.

KAnedA, Y. \& MorishitA, K. 2013 Small-scale statistics and structure of turbulence - in the light of high resolution direct numerical simulation. In Ten Chapters in Turbulence (ed. P. A. Davidson, Y. Kaneda \& K. R. Sreenivasan), pp. 1-42. Cambridge University Press.

Kolmogorov, A. N. 1941a Dissipation of energy under locally isotropic turbulence. Dokl. Akad. Nauk SSSR 32, 16-18.

Kolmogorov, A. N. $1941 b$ The local structure of turbulence in an incompressible viscous fluid for very large Reynolds numbers. Dokl. Akad. Nauk SSSR 30, 301-305.

Kolmogorov, A. N. 1962 A refinement of previous hypotheses concerning the local structure of turbulence in a viscous incompressible fluid at high Reynolds number. J. Fluid Mech. 13, $82-85$.

Kurien, S. \& SReenivasan, K. R. 2001 Dynamical eqautions for high-order structure functions and a comparison of a mean-field theory with experiments on three-dimensional turbulence. Phys. Rev. E 64, 056302.

Landau, L. D. \& Lifshitz, E. M. 1959 Fluid Mechanics. Pergamon.

L'vov, V. \& Procaccia, I. $1996 a$ Fusion rules in turbulent systems with flux equilibrium. Phys. Rev. Lett. 76 (16), 2898-2901.

L'vov, V. \& PROCACCIA, I. $1996 b$ Towards a nonperturbative theory of hydrodynamic turbulence: fusion rules, exact bridge relations, and anomalous viscous scaling functions. Phys. Rev. E 54 (6), 6268-6284.

Meneveau, C. \& SReenivasan, K. R. 1991 The multifractional nature of turbulent energy dissipation. J. Fluid Mech. 224, 429-484.

Nakano, T., Gotoh, T. \& Fukayama, D. 2003 Roles of convection, pressure, and dissipation in three-dimensional turbulence. Phys. Rev. E 67, 026316.

Nelkin, M. 1994 Universality and scaling in fully developed turbulence. Adv. Phys. 43, 81-143.

Oвukhov, A. R. 1962 Some specific features of atmospheric turbulence. J. Fluid Mech. 13, 77-81.

Polyakov, A. M. 1995 Turbulence without pressure. Phys. Rev. E 52 (6), 6183-6188.

Rogallo, R. S. 1981 Numerical experiments in homogeneous turbulence. NASA Tech. Mem. 81315.

She, Z. S. \& LÉvÊQUe, E. 1994 Universal scaling laws in fully developed turbulence. Phys. Rev. 72, 336-339.

She, Z. S. \& Waymire, E. C. 1995 Quantitized energy cascade and log-Poisson statistics in fully developed turbulence. Phys. Rev. Lett. 74, 262-265.

Siggia, E. D. 1981 Invariants for the one-point vorticity and strain rate correlation functions. Phys. Fluids 24, 1934-1936.

Sreenivasan, K. R. \& Antonia, R. A. 1997 The phenomenology of small-scale turbulence. Annu. Rev. Fluid Mech. 29, 435-472.

Stolovitzky, G., Kailasnath, P. \& Sreenivasan, K. R. 1992 Kolmogorov's refined similarity hypotheses. Phys. Rev. Lett. 69 (8), 1178-1181. 
YAкнот, V. 2001 Mean-field approximation and a small parameter in turbulence theory. Phys. Rev. E 63, 026307.

Yакнот, V. 2003 Pressure-velocity correlations and scaling exponents in turbulence. J. Fluid Mech. 495, 135-143.

YAKHot, V. 2006 Probability densities in strong turbulence. Physica D 215 (2), 166-174.

YAKHOT, V. \& SREENIVASAN, K. R. 2005 Anomalous scaling of structure functions and dynamic constraints on turbulence simulations. J. Stat. Phys. 121, 823-884. 